Article

\title{
Classical and Quantum Models in Non-Equilibrium Statistical Mechanics: Moment Methods and Long-Time Approximations
}

\author{
Ramon F. Alvarez-Estrada \\ Departamento de Fisica Teorica I, Facultad de Ciencias Fisicas, Universidad Complutense, \\ 28040 Madrid, Spain; E-Mail: rfa@ fis.ucm.es; Tel.: +34-91-3944595
}

Received: 21 December 2011; in revised form: 25 January 2012 / Accepted: 9 February 2012 /

Published: 15 February 2012

\begin{abstract}
We consider non-equilibrium open statistical systems, subject to potentials and to external "heat baths" $(h b)$ at thermal equilibrium at temperature $T$ (either with ab initio dissipation or without it). Boltzmann's classical equilibrium distributions generate, as Gaussian weight functions in momenta, orthogonal polynomials in momenta (the position-independent Hermite polynomials $H_{n}$ 's). The moments of non-equilibrium classical distributions, implied by the $H_{n}$ 's, fulfill a hierarchy: for long times, the lowest moment dominates the evolution towards thermal equilibrium, either with dissipation or without it (but under certain approximation). We revisit that hierarchy, whose solution depends on operator continued fractions. We review our generalization of that moment method to classical closed many-particle interacting systems with neither a $h b$ nor ab initio dissipation: with initial states describing thermal equilibrium at $T$ at large distances but non-equilibrium at finite distances, the moment method yields, approximately, irreversible thermalization of the whole system at $T$, for long times. Generalizations to non-equilibrium quantum interacting systems meet additional difficulties. Three of them are: (i) equilibrium distributions (represented through Wigner functions) are neither Gaussian in momenta nor known in closed form; (ii) they may depend on dissipation; and (iii) the orthogonal polynomials in momenta generated by them depend also on positions. We generalize the moment method, dealing with (i), (ii) and (iii), to some non-equilibrium one-particle quantum interacting systems. Open problems are discussed briefly.
\end{abstract}

Keywords: classical and quantum distributions; equilibrium solutions and orthogonal polynomials; non-equilibrium moments; long-time approximations; approximate irreversibility 
PACS Classification: 05.20.-y, 05.10.Gg, 05.20.Jj, 05.30.-d

\section{Introduction}

A quite wide and very interesting set of references, from different standpoints, related to and/or oriented towards the foundations of non-equilibrium Statistical Mechanics can be seen in [1]. Non-equilibrium statistical systems of classical particles are described by Liouville classical distribution functions $\left(W_{c}\right)$ [2-5]. For non-equilibrium statistical systems of quantum particles, several distribution functions are available, Wigner functions $(W)$ [3-7] being a suitable and simple possibility in a global sense (in spite of the fact that their positivity is not warranted). Open statistical systems (involving, typically, one or a few degrees of freedom) are those subject to external "heat baths" $(h b)$ or reservoirs at thermal equilibrium at temperature $T$, with finite or small dissipation due to the $h b$. We shall regard vanishing dissipation as a mathematical idealization of the case with small friction. Closed statistical systems are those not subject to any external $h b$, and they are formed by an enormously large number of interacting particles. General equations describing, in differential form, the time evolutions of non-equilibrium closed interacting classical or quantum systems (with some initial off-equilibrium state) are well known [2-5]. On the other hand, approximate time evolution differential equations for the effective or relevant degrees of freedom for non-equilibrium open or closed (classical or quantum) interacting systems are known in certain cases [2-4,6,7]. Generically, those evolution equations turn out to be increasingly difficult to solve (even on a computer), as the number of particles in the system grows and/or one tries to describe the state of the system for increasingly long times (starting from some initial non-equilibrium state). On the other hand, there does exist certain global understanding of various classes of solutions of those evolution equations, at least in a number of cases. Anyway, to achieve wider and better knowledge of how open or closed (classical or quantum) statistical interacting systems evolve in time continues to play a key role in Statistical Physics. In particular, an issue of crucial importance is how irreversibility could arise in the long-time evolution of closed classical or quantum many-particle systems $[2-5,8]$, or stated in other words, how an arrow of time could set in.

Non-equilibrium open one-particle statistical classical systems, subject to potentials and to dissipation due to the $h b$, lend themselves typically to perform certain constructions and approximations in a framework which is simpler than those met for other cases: see [9-12] and references therein. We shall remind them [9-12] firstly, in order to motivate our developments. In them, classical time-independent equilibrium distributions $\left(W_{c, e q}\right)$, which are independent on dissipation, have been used as weight functions to generate families of orthogonal polynomials in momenta. The latter has been possible due to two distinguishing and simplifying features of $W_{c, e q}$ : dependences on classical momenta and those on spatial coordinates factorize, and $W_{c, e q}$ is Gaussian in classical momenta. Then, the orthogonal polynomials generated by $W_{c, e q}$ turned out to be just the standard Hermite polynomials [13] and, moreover, they are independent on spatial coordinates (and on dissipation). Those orthogonal polynomials yield (by integrating over classical momenta) moments $W_{c, n}$ of the non-equilibrium classical distributions $W_{c}$. The non-equilibrium $W_{c, n}$ 's depend on spatial coordinates and time $(t)$ and 
on dissipation (but not on momenta), and fulfill an infinite linear hierarchy. A key issue was that, for long times, the lowest moment appears to dominate the evolution towards thermal equilibrium with the $h b$ (and, hence, to become independent on the initial non-equilibrium state and on dissipation) $[9,10]$.

Interesting open issues in [9-12], among others, include: (a) further study of the process leading to moments which depend only on spatial coordinates; (b) properties of the solutions of the non-equilibrium classical hierarchies for different potentials (vanishing or not at large distances) and their behaviour for long times; (c) generalizations to non-equilibrium closed classical interacting many-particle systems without external $h b$ 's (with potentials vanishing at large distances), with initial states at thermal equilibrium at temperature $T$ at large distances but off-equilibrium at finite distances and with the possibility of obtaining, in a suitable long-time approximation, irreversibility; (d) generalizations of (c) when classical electromagnetic degrees of freedom are included; (e) extensions to open one-particle quantum systems by means of Wigner functions (either with or without dissipation); (f) closed quantum interacting many-particle systems, which clearly lead to far more difficult problems. The above non-equilibrium moment method relies, very crucially, on the previous knowledge and properties of equilibrium distributions. Being natural to work with quantum Wigner functions (although not mandatory), we anticipate certain genuine quantum difficulties: the equilibrium quantum Wigner functions are neither Gaussian in momenta nor known in closed form, and dependences on momenta and those on spatial coordinates do not factorize, in general. The issue of the possible dependence of the quantum equilibrium distributions on dissipation leads to additional complications. The use of other quantum distribution functions does not appear to yield improvements in a global sense.

We shall review in outline items (a)-(c), analyzed in our previous work [14-19]. We shall study further convergence aspects for issues (b) and (c), which, even if shortly, will provide some additional partial clarification, so far unpublished. We shall present a simple overview of [14] on long-time approximations and irreversibility in closed interacting many-particle classical systems, including some improvements from [16] and [18,19]. Item (d) has been dealt with in [15] in detail and we shall not add more here. And, finally, we shall concentrate on (e). The genuine difficulties of quantum cases anticipated above already show up in one-dimensional models. Some generalizations of the moment method to non-equilibrium open one-particle quantum interacting systems, without or with dissipation and to lowest order in Planck's constant, have already been studied [18,20]. Here, we shall concentrate on constructing the equilibrium Wigner functions, the families of orthogonal polynomials generated by the latter and non-equilibrium moments and equations at low order in the hierarchies, to all orders in Planck's constant, in one-dimensional models. Models in which the equilibrium Wigner functions either depend on dissipation or are independent on it will be analyzed. Item (f) above (closed many-particle interacting quantum systems and irreversibility issues) will not be addressed in this work.

This paper is organized as follows. Section 2 reviews open one-dimensional classical systems with or without dissipation. Section 3 treats classical closed interacting many-particle systems. Section 4 deals with general aspects of quantum-mechanical one-dimensional models, in the idealized limit of vanishing dissipation. Section 5 is devoted to open quantum-mechanical one-dimensional models with quartic plus quadratic potential, also without dissipation, through an approach different from that in Section 4. Section 6 treats open quantum-mechanical one-dimensional models with quadratic plus quartic potential with dissipation. Various technical aspects in Sections 4, 5 and 6 are treated in 
Appendices A-D. Finally, Section 7 presents some conclusions and various discussions. A short account of the contents of Sections 5 and 6 has been presented orally in the 11th International Conference on Orthogonal Polynomials, Special Functions and Applications, held in Universidad Carlos III, Madrid, Spain (August 29 through September 2, 2011).

\section{Open Classical One Particle Systems}

\subsection{One-Dimensional Case: Some General Aspects}

Let a classical particle, with mass $m$, position $x$ and momentum $q$, be subject to a real potential $V=V(x)$, in the presence of a $h b$ at thermal equilibrium at temperature $T$. We shall employ the standard variable $\beta=\left(k_{B} T\right)^{-1}$ ( $k_{B}$ being Boltzmann's constant). To simplify matters, the potential will be supposed to be repulsive: $V(x) \geq 0$ : either $V(x) \rightarrow 0$ as $|x| \rightarrow+\infty$ (Subsection 2.3) or $V(x) \equiv 0$ (Subsection 2.4) or $V(x)$ will correspond to a harmonic oscillator (Subsections 2.5). The classical Hamiltonian of the particle is: $H_{c}=q^{2} /(2 m)+V$. Let the classical particle be, at the initial time $t=0$, out of thermal equilibrium with the $h b$, and have a probability distribution $W_{c, \text { in }}=W_{c, \text { in }}(x, q)$ $(\geq 0)$ to be at the position $x$ with momentum $q$. Then, the non-equilibrium particle could be, at time $t(>0)$, at the position $x$ with momentum $q$, with probability distribution $W_{c}=W_{c}(x, q ; t)(\geq 0)$. For instance, the $h b$ could be air (at rest and at thermal equilibrium, at $T \simeq 300 \mathrm{~K}$ ) in a room, and the classical particle could be a virus or a grain of pollen, performing Brownian motion in air.

How does $W_{c}$ evolve in time? A temporal evolution, including nonvanishing dissipation effects on the particle due to the $h b$ (described, in turn, by the friction constant $\sigma>0$ ), is provided by the irreversible Kramers equation [9-12]:

$$
\frac{\partial W_{c}}{\partial t}+\frac{q}{m} \frac{\partial W_{c}}{\partial x}-\frac{\partial V}{\partial x} \frac{\partial W_{c}}{\partial q}=\frac{1}{\sigma} \frac{\partial\left[(q+(m / \beta))\left(\partial W_{c} / \partial q\right)\right]}{\partial q}
$$

The equilibrium (or Boltzmann's) canonical distribution, the $t$-independent solution of Equation (1) describing thermal equilibrium of the particle with the $h b$, is: $W_{c}=W_{c, e q}=\exp \left[-\beta\left(q^{2} /(2 m)+V\right)\right]$. The physics involved in the characterization of the equilibrium distribution (to be reached for very long $t$ ), which constitutes the core of Equilibrium Classical Statistical Mechanics, selects uniquely $W_{c, e q}$. Notice that $W_{c, e q}$ is $\sigma$-independent and, hence, it is not influenced by the dissipation mechanism embodied in the right-hand-side of Equation (1). The last property does not hold necessarily in the quantum case.

For another time evolution, let dissipation effects on the particle due to the $h b$ be supposed so small that they are discarded completely (vanishing friction). Then, the time evolution of $W_{c}$ is given by the reversible Liouville equation:

$$
\frac{\partial W_{c}}{\partial t}+\frac{q}{m} \frac{\partial W_{c}}{\partial x}-\frac{\partial V}{\partial x} \frac{\partial W_{c}}{\partial q}=0
$$

$W_{c, e q}$ is a $t$-independent solution of Equation (2). After some long-time approximation, $W_{c} \rightarrow W_{c, e q}$ in some sense: see Subsection 2.3.

Let $H_{n}(y)$ be the standard $n$-th Hermite polynomial [13]. We remind that the $H_{n}(y)$ 's constitute an infinite family of orthogonal polynomials in $y$, with the weight $\exp \left[-y^{2}\right]$ : $\int_{-\infty}^{+\infty} d y \exp \left[-y^{2}\right] H_{n}(y) H_{n^{\prime}}(y)=\pi^{1 / 2} 2^{n} n ! \delta_{n, n^{\prime}}, \delta_{n, n^{\prime}}$ being the Kronecker delta symbol $\left(\delta_{n, n^{\prime}}=0,1\right.$, 
if $n \neq n^{\prime}, n=n^{\prime}$, respectively) [13]. For both Equations (1) and (2), we shall introduce the (normalized) non-equilibrium classical moments $W_{c, n}=W_{c, n}(x ; t)(n=0,1,2, \ldots)$ of $W_{c}[9-12,14,16]$ :

$$
W_{c, n}=\int_{-\infty}^{+\infty} d\left(q / q_{e q}\right) \frac{H_{n}\left(q / q_{e q}\right)}{\left(\pi^{1 / 2} 2^{n} n !\right)^{1 / 2}} W_{c}(x, q ; t), q_{e q}=(2 m / \beta)^{1 / 2}
$$

$W_{c, 0}$ is the marginal probability distribution for $x$. If $W_{c}=W_{c, e q}$, then $W_{c, e q, 0}$ is proportional to $\exp [-\beta V]$ and $W_{c, e q, n}=0$ for $n=1,2, \ldots$ Equation (3) can also be applied to the initial off-equilibrium distribution $W_{c, i n}$ and gives the initial moments, $W_{c, i n, n}$. It will be very convenient to work also with the symmetrized moments $g_{n}=W_{c, e q, 0}^{-1 / 2} W_{c, n}$. The time evolution of $g_{n}$ will be treated below.

\subsection{Classical Non-Equilibrium and Formal Solution Using Operator Continued Fractions}

Equations (1) and (3) yield the infinite irreversible three-term linear hierarchy for $g_{n}$ 's $\left(n=0,1,2, \ldots, g_{-1}=0\right)[9-12,20]$ :

$$
\begin{aligned}
\frac{\partial g_{n}}{\partial t} & =-M_{n, n+1} g_{n+1}-M_{n, n-1} g_{n-1}-\frac{n}{\sigma} g_{n} \\
M_{n, n \pm 1} W_{c, s, n \pm 1} & \equiv\left[\frac{(n+(1 / 2)(1 \pm 1)) K_{B} T}{m}\right]^{1 / 2}\left[\frac{\partial W_{c, s, n \pm 1}}{\partial x}-\frac{( \pm 1) W_{c, s, n \pm 1}}{2 K_{B} T} \frac{d V}{d x}\right]
\end{aligned}
$$

On the other hand, Equations (2) and (3) yield the infinite reversible three-term linear hierarchy for $g_{n}$ 's ( $n=0,1,2, \ldots, g_{-1}=0$ ) [14,16], which is the same as in Equation (4), with $\sigma^{-1}=0$. For the hierarchy Equation (4) and for the reversible one, with $\sigma^{-1}=0$, the initial condition is formed by the set of all $W_{c, e q, 0}^{-1 / 2} W_{c, i n, n}$. We shall treat both $\sigma^{-1}>0$ and $\sigma^{-1}=0$ simultaneously, unless otherwise stated.

The $M_{n, n \pm 1}$ 's are linear first-order differential operators, none of which is Hermitian: except for the $n$-dependent factors, their structures are typical of those appearing in the study of the Smoluchowski equation [10]. $M_{n, n+1}$ is the adjoint of $-M_{n+1, n}$. Notice that, if $(d V / d x) \neq 0, M_{n, n-1}$ does not commute with $M_{n, n+1}$. Let us consider the Laplace transforms $\tilde{W}_{c, n}=\tilde{W}_{c, n}(s)=\int_{0}^{+\infty} d t W_{c, n} \exp (-s t)$. with inverse $W_{c, n}=\int_{c-i \infty}^{c+i \infty}(d s / 2 \pi i) \tilde{W}_{c, n} \exp (s t)$ ( $c$ being real and such that $\tilde{W}_{c, n}(s)$ is analytic in the half-plane Res $>c$ of the complex $s$-plane). It will also be useful to deal with the symmetrized Laplace transforms $\tilde{g}_{n}=W_{c, e q, 0}^{-1 / 2} \tilde{W}_{c, n}$. This definition and Equation (4) yield the symmetrized three-term hierarchy for $\tilde{g}_{n}$ :

$$
\left(s+\frac{n}{\sigma}\right) \tilde{g}_{n}=W_{c, e q, 0}^{-1 / 2} W_{c, i n, n}-M_{n, n+1} \tilde{g}_{n+1}-M_{n, n-1} \tilde{g}_{n-1}
$$

for both $\sigma^{-1}>0$ (irreversible) and $\sigma^{-1}=0$ (reversible).

The hierarchy Equation (6) can be solved formally by extending to it standard procedures for solving numerical three-term linear recurrence relations in terms of continued fractions (see, for instance, [10]). Thus, one neglects $\tilde{g}_{n^{\prime}+1}(s)$ in Equation (6) for given $n^{\prime}$, solves for $\tilde{g}_{n^{\prime}}(s)$ in terms of $\tilde{g}_{n^{\prime}-1}(s)$, proceeds to Equation (6) for $n^{\prime}-1$, solves for $\tilde{g}_{n^{\prime}-1}(s)$ in terms of $\tilde{g}_{n^{\prime}-2}(s)$ and so on. Then, one infers directly the general formal (continued-fraction) structure of the solution as $n^{\prime} \rightarrow+\infty$. That formal procedure yields all $\tilde{g}_{n}(s)$, for any $n=1, \ldots$, in terms of sums of products of certain $s$-dependent linear operators $D\left[n^{\prime} ; s+\frac{n^{\prime}}{\sigma}\right], n^{\prime} \geq n$, acting upon $\tilde{g}_{n-1}(s)$ and upon all $W_{c, e q, 0}^{-1 / 2} W_{c, i n, n^{\prime}}$ s, with $n^{\prime} \geq n$. The linear operators $D\left[n ; s+\frac{n}{\sigma}\right]$ 's are defined recurrently through:

$$
D\left[n ; s+\frac{n}{\sigma}\right]=\left[s I+\frac{n}{\sigma}-M_{n, n+1} D\left[n+1 ; s+\frac{n+1}{\sigma}\right] M_{n+1, n}\right]^{-1}
$$


$I$ is the unit operator. By iteration of Equation (7), $D\left[n ; s+\frac{n}{\sigma}\right]$ becomes a formal infinite continued fraction of products of the linear operators $M_{n, n+1}$ and $M_{n+1, n}$ (which do not commute if $d V / d x \neq 0$ ).

For a simpler hierarchy and a clearer exposition, without loss of generality, let us assume that $W_{c, i n, n^{\prime}}=0$ for $n^{\prime} \geq 1$, with $W_{c, i n, 0} \neq 0$ (and $\left.\neq W_{c, e q, 0}\right)$. Also, let us choose some $n_{0}(\geq 1)$. Then, Equation (6) yields:

$$
\begin{aligned}
\left(s+\frac{n}{\sigma}\right) \tilde{g}_{n} & =W_{c, e q, 0}^{-1 / 2} W_{c, i n, 0} \delta_{n, 0}-M_{n, n+1} \tilde{g}_{n+1}-M_{n, n-1} \tilde{g}_{n-1}, & & n \leq n_{0}-1 \\
\tilde{g}_{n}(s) & =-D\left[n ; s+\frac{n}{\sigma}\right] M_{n, n-1} \tilde{g}_{n-1}(s), & & n \geq n_{0}
\end{aligned}
$$

$\delta_{n, 0}$ is the Kronecker delta symbol. If $\sigma^{-1}=0$ and $n_{0}=1$, Equations (8) and (9) yield:

$$
\tilde{g}_{0}(s)=D[0 ; s] W_{c, e q, 0}^{-1 / 2} W_{c, i n, 0}
$$

It should be clear that if $\sigma^{-1}=0$ (absence of dissipation) the particle is not expected to reach irreversibly, for long time, thermal equilibrium with the $h b$. In such a case, Equation (10), in which no long-time approximation has been carried out so far, is as reversible as Equation (2).

\subsection{Operator Continued Fractions and Long-Time Approximation}

We continue with both $\sigma^{-1}>0$ and $\sigma^{-1}=0$, unless otherwise stated. Let us choose $n(\geq 1)$ and fix $s=\epsilon>0$ (real and suitably small) in any $D\left[n ; s+\frac{n}{\sigma}\right]$. Then, the following crucial properties appear to hold [14,15]: if $D\left[n+1 ; \epsilon+\frac{n+1}{\sigma}\right]$ were Hermitian and if all its eigenvalues (which would be real) were non-negative, then the same would hold true for $D\left[n ; \epsilon+\frac{n}{\sigma}\right]$. It is easy to confirm the validity of that property if $D\left[n+1 ; \epsilon+\frac{n+1}{\sigma}\right]$ is replaced by a $2 \times 2$ matrix with the above (non-negativity and Hermiticity) properties and $M_{n, n+1}$ and $M_{n+1, n}$ are chosen as $2 \times 2$ matrices such that $M_{n, n+1}$ be the adjoint of $-M_{n+1, n}$. A confirming example with a $2 \times 2$ matrix is given in Appendix D in [15]. Then, through iterative arguments, $D\left[n ; \epsilon+\frac{n}{\sigma}\right]$ 's, $n=0,1,2,3, \ldots$, are Hermitian, and all their eigenvalues are non-negative. These properties will be crucial for the long-time approximation below.

The long-time approximation for $n \geq n_{0} \geq 1$ reads as follows. One replaces any $D\left[n ; s+\frac{n}{\sigma}\right]$ yielding $\tilde{g}_{n}$ in Equation (9), $n \geq n_{0}$, by $D\left[n ; \epsilon+\frac{n}{\sigma}\right]$ : this approximation is not done for $n<n_{0}$, which will be crucial, and is the better, the larger $n_{0}$. We regard $D\left[n_{0} ; \epsilon+\frac{n_{0}}{\sigma}\right]$ in Equation (9) for $n=n_{0}$ as a fixed ( $s$-independent) operator. Then, for small $s$, we approximate in Equation (9) for $n=n_{0}$ as: $\tilde{g}_{n_{0}}(s) \simeq-D\left[n_{0} ; \epsilon+\frac{n_{0}}{\sigma}\right] M_{n_{0}, n_{0}-1} \tilde{g}_{n_{0}-1}(s)$ [to be compared to Equation (9)]. The resulting hierarchy Equation (8) for $\tilde{g}_{n}$ 's $\left(n=0, \ldots, n_{0}-1\right)$, through the inverse Laplace transform, yields formally a closed approximate irreversible hierarchy for $g_{n}, n=0,1, \ldots, n_{0}-1$. In the latter closed hierarchy, after the above long-time approximation, we choose to employ the same initial condition $W_{c, i n, 0}$ at $t=0$ : this amounts to another kind of approximation. The solutions of the last closed hierarchy for $g_{n}$ relax irreversibly, for large $t$ and reasonable $W_{c, i n, 0}$, towards $W_{c, e q, 0}^{1 / 2} \neq 0$ for $n=0$ and 0 if $n=1, \ldots, n_{0}-1$ (thermal equilibrium) $[14,15]$. Then, for long-time, the dominant moment is $g_{0}$, while any $g_{n}$ with $n>0$ is negligible. $g_{n}\left(n \geq n_{0}\right)$ is the smaller, the larger $t(>0)$ and $n$ (due to the behaviour of $D\left[n ; \epsilon+\frac{n}{\sigma}\right]$ with $n$ ). Similar behaviours hold for $W_{c, 0}$ and $W_{c, n}$ with $n>0$. To carry out quantitative studies for large t, some ansatz or approximation should be provided for $D\left[n_{0} ; \epsilon+\frac{n_{0}}{\sigma}\right]$, consistent with the above properties. 
We shall illustrate the above facts by taking, for simplicity, $n_{0}=1$ (with either $\sigma^{-1}>0$ or $\sigma^{-1}=0$ ). Then, Equations (9) (with the above approximation) and (8) yield, by taking inverse Laplace transforms:

$$
\begin{aligned}
\frac{\partial g_{0}}{\partial t} & =-M_{0,1} g_{1} \\
g_{1} & \simeq-D\left[1 ; \epsilon+\sigma^{-1}\right] M_{1,0} g_{0}
\end{aligned}
$$

Equations (11) and (12) give the irreversible Smoluchowski-like equation for the $n=0$ moment:

$$
\partial g_{0} / \partial t=M_{0,1} D\left[1 ; \epsilon+\sigma^{-1}\right] M_{1,0} g_{0}
$$

with initial condition $W_{c, e q, 0}^{-1 / 2} W_{c, i n, 0}$. For the computations below, we shall interpret the right-hand-side of Equation (13) as $\int_{-\infty}^{+\infty} d x^{\prime}\left(M_{0,1} D\left[1 ; \epsilon+\sigma^{-1}\right] M_{1,0}\right)\left(x, x^{\prime}\right) g_{0}\left(x^{\prime}\right)$. Let: $\left(f_{1}, f_{2}\right)=\int_{-\infty}^{+\infty} d x f_{1}(x)^{*} f_{2}(x)$ for suitable functions $f_{1}$ and $f_{2}$. Due to the Hermiticity of $D\left[1 ; \epsilon+\sigma^{-1}\right]:\left(f_{1}, M_{0,1} D\left[1 ; \epsilon+\sigma^{-1}\right] M_{1,0} f_{2}\right)=$ $\left(M_{0,1} D\left[1 ; \epsilon+\sigma^{-1}\right] M_{1,0} f_{1}, f_{2}\right)$, thereby checking that $M_{0,1} D\left[1 ; \epsilon+\sigma^{-1}\right] M_{1,0}$ is Hermitian. Moreover: $\left(f_{1}, M_{0,1} D\left[1 ; \epsilon+\sigma^{-1}\right] M_{1,0} f_{1}\right)=-\left(M_{1,0} f_{1}, D\left[1 ; \epsilon+\sigma^{-1}\right] M_{1,0} f_{1}\right) \leq 0$ for arbitrary functions $f_{1}$, as all eigenvalues of $D\left[1 ; \epsilon+\sigma^{-1}\right]$ are $\geq 0$. Let $f_{\lambda}(x)$ be an eigenfunction of the integral operator $M_{0,1} D[1 ; \epsilon+$ $\left.\sigma^{-1}\right] M_{1,0}$ with eigenvalue $\lambda(\leq 0)$. Then, $\left(M_{0,1} D\left[1 ; \epsilon+\sigma^{-1}\right] M_{1,0}\right)\left(x, x^{\prime}\right)=\sum_{\lambda} \lambda f_{\lambda}(x) f_{\lambda}\left(x^{\prime}\right)^{*} . \sum_{\lambda}$ is a short-hand notation denoting integration over the whole spectrum of $M_{0,1} D\left[1 ; \epsilon+\sigma^{-1}\right] M_{1,0}$. By expanding $W_{c, e q, 0}^{-1 / 2} W_{c, i n, 0}=\sum_{\lambda} g_{i n, \lambda} f_{\lambda}(x)$, with $x$-independent $g_{i n, \lambda}$, the solution of Equation (13) with the above initial condition is $g_{0}=\sum_{\lambda} g_{i n, \lambda} f_{\lambda}(x) \exp \lambda t$, which relaxes irreversibly as $t \rightarrow+\infty$ towards $g_{i n, 0} f_{0}(x)$, corresponding to $\lambda=0$. At equilibrium, one has: $g_{0}=W_{c, e q, 0}^{1 / 2}$ (proportional to $\left.f_{0}\right), M_{1,0} g_{0}=0$ and $g_{n}=0, n=1,2,3, \ldots$, consistently with [9,10]. Let us restrict the above to $\sigma^{-1}=0$. Then, Equation (13) with $\sigma^{-1}=0$ is (at least, with $\epsilon>0$ ) as irreversible as the standard heat equation: for long $t(>0)$ the dominant moment is $g_{0}$, while any $g_{n}$ with $n>0$ is negligible, $g_{n}$ being the smaller, the larger $n$ and $t>0)$, and so on for the $W_{c, n}$ 's. As stressed after Equation (10), irreversible thermalization does not occur if $\sigma^{-1}=0$, in the absence of long-time approximations. Then, for $\sigma^{-1}=0$, to have carried out mathematical approximations (say, $D[n ; s] \simeq D[n ; \epsilon]$ for $n \geq n_{0}$, but not for $n<n_{0}$ ), which give rise to thermalization with the $h b$ [like that based on Equation (13)], could be regarded as an alternative way of introducing irreversibility out of a reversible model [namely, Equation (2)] with vanishing dissipation ab initio. The latter interpretation with $\sigma^{-1}=0$ is consistent for the one-dimensional case. We shall regard it as a mathematical introduction, which will be very helpful to deal later with non-equilibrium classical closed interacting many-particle systems without external $h b$ 's.

\subsection{Convergence Properties for $V \equiv 0$ and $\sigma^{-1}=0$}

We shall now undertake a more detailed analysis for $\sigma^{-1}=0$ and $V \equiv 0$, in order to understand the structure and convergence of the operator continued fractions $D[n ; s]$ generated by Equation (7). To fix the ideas, we shall take $\epsilon>0$ first, so as to allow for $\epsilon \rightarrow 0$ later, thereby reviewing and extending [14]. Equation (13) becomes formally (using standard notations for continued fractions [21]):

$$
\begin{aligned}
\partial g_{0} / \partial t & =\frac{k_{B} T}{m} \frac{\partial}{\partial x} D[1 ; \epsilon] \frac{\partial}{\partial x} g_{0} \\
D[1 ; \epsilon] & =\frac{1}{\epsilon I+} \frac{2\left[\left(k_{B} T / m\right)\left(-\partial^{2}\right) /\left(\partial x^{2}\right)\right]}{\epsilon I+} \frac{3\left[\left(k_{B} T / m\right)\left(-\partial^{2}\right) /\left(\partial x^{2}\right)\right]}{\epsilon I+} \cdots
\end{aligned}
$$


Let us perform a spatial Fourier transformation from configuration space $(x)$ to wavevector space $(k)$, by applying $(2 \pi)^{-1 / 2} \int d x \exp (-i k x)$. Let $e(k) \equiv(2 m)^{-1} k_{B} T k^{2}$. Then, the Fourier transforms of the operator continued fractions in Equation (7), for $\operatorname{Re} s>0$ and $n \geq 0$, and in Equation (14) are:

$$
\begin{aligned}
D_{1}[k ; n ; s] & =\left[s+2(n+1) e(k ; N) D_{1}[k ; n+1 ; s]^{-1}\right. \\
\partial g_{0,1} / \partial t & =i\left(k_{B} T / m\right)^{1 / 2} k D_{1}[k ; 1 ; \epsilon] i\left(k_{B} T / m\right)^{1 / 2} k g_{0,1}
\end{aligned}
$$

$g_{0,1}=g_{0,1}(k, t)$ being the spatial Fourier transform of $g_{0}$. By iteration, $D_{1}[k ; n ; s]$ becomes the following ordinary continued fraction (in standard notations [21]):

$$
D_{1}[k ; n ; s]=\frac{1}{s+} \frac{2 e(k)(n+1)}{s+} \frac{2 e(k)(n+2)}{s+} \cdots=\frac{1}{e(k)^{1 / 2}} \cdot\left[\frac{2^{-1}}{z+} \frac{2^{-1}(n+1)}{z+} \frac{2^{-1}(n+2)}{z+} \cdots\right]
$$

with $z=s /\left(2 e(k)^{1 / 2}\right)$. For real $s \geq 0, D_{1}[k ; n ; s]$ is real. On the other hand:

$$
\frac{2^{-1}}{z+} \frac{2^{-1}(n+1)}{z+} \frac{2^{-1}(n+2)}{z+} \cdots=\frac{i^{n} \operatorname{erfc}(z)}{i^{n-1} \operatorname{erfc}(z)}
$$

with $i^{n} \operatorname{erf} c(z)=\int_{z}^{+\infty} d z^{\prime} i^{n-1} \operatorname{erf} c\left(z^{\prime}\right), i^{0} \operatorname{erfc}(z)=\operatorname{erfc}(z)$ (the complementary error function) and $i^{-1} \operatorname{erfc}(z)=\left(2 / \pi^{1 / 2}\right) \exp \left(-z^{2}\right)$ [21]. One has: $D_{1}[k ; n ; \epsilon] \geq 0$. With $\epsilon>0$ and by using [21], one gets: $D_{1}[k ; 1 ; \epsilon] \rightarrow \epsilon^{-1}$ as $k \rightarrow 0$, while $D_{1}[k ; 1 ; \epsilon] \rightarrow(\pi e(k))^{-1 / 2}$ as $|k| \rightarrow \infty$. For $s=0$, the behaviour of $D_{1}$ is different. Equation (18) gives:

$$
D_{1}[k ; n ; s=\epsilon=0]=D_{1}[k ; n ; 0]=\left[2 e(k)^{1 / 2}\right]^{-1} \frac{1}{0+} \frac{2^{-1}(n+1)}{0+} \frac{2^{-1}(n+2)}{0+} \cdots
$$

The continued fraction in Equation (20) can, in turn, be evaluated in terms of the standard Gamma function $\Gamma[21]$ :

$$
\frac{2^{-1}}{0+} \frac{2^{-1}(n+1)}{0+} \frac{2^{-1}(n+2)}{0+} \cdots=\frac{\Gamma((n / 2)+1 / 2)}{2 \Gamma((n / 2)+1)}>0, n \geq 1
$$

For large $n$, the ratio in Equation $(21) \rightarrow n^{-1 / 2}$. Equation (19) behaves similarly. For $k \rightarrow 0, D_{1}[k ; n ; 0]$ diverges as $k^{-1}$ (due to $e(k)^{-1 / 2}$ ). Then, $\int d k D_{1}[k ; n ; 0]$ also diverges near $k=0$.

\subsection{Classical Harmonic Oscillator: Operator Continued Fractions}

For vanishing dissipation due to the $h b$, which is at thermal equilibrium at $T, D[n ; s]$ has also been evaluated for a classical harmonic oscillator $\left(V=2^{-1} m \omega^{2} x^{2}\right.$ with frequency $\left.\omega>0\right)$ in one space dimension [16]. It will be methodologically adequate to outline those results here. The actual classical hierarchy turns out to be exactly solvable, by inspiring on known algebra for the quantum harmonic oscillator: compare with [10]. Here, we shall work with the dimensionless position and momentum variables $y \equiv\left[\left(2 k_{B} T\right)^{-1} m\right]^{1 / 2} \omega x, \pi \equiv\left[\left(2 k_{B} T m\right)^{-1}\right]^{1 / 2} q$. Accordingly, we shall deal with $W_{c}(x . q ; t)=$ $f(y, \pi ; t)=f$. The non-equilibrium moments for $f$ are introduced through Equation (3), with the corresponding changes. For convenience, we introduce, in the actual classical context, "annihilation" (a) and "creation" $\left(a^{+}\right)$operators: $a \equiv 2^{-1 / 2}(d / d y+y), a^{+} \equiv 2^{-1 / 2}(-d / d y+y)$, with commutator $\left[a, a^{+}\right]=+1([A, B] \equiv A B-B A)$. Notice that $a^{+}$and $a$ are proportional to $M_{n, n+1}$ and $M_{n, n-1}$, 
respectively. We proceed like in Subsections 2.1 and 2.2. Then, the hierarchy for the symmetrized $\tilde{g}_{n}(s)\left(=\tilde{g}_{n}(y, s)\right.$ ), which is the actual counterpart of Equation (6), becomes (with $\sigma^{-1}=0$ ):

$$
s \tilde{g}_{n}=W_{c, e q, 0}^{-1 / 2} W_{c, i n, n}+\omega\left[(n+1)^{1 / 2} \cdot a^{+} \tilde{g}_{n+1}-n^{1 / 2} \cdot a \tilde{g}_{n-1}\right]
$$

with the initial condition $W_{c, e q, 0}^{-1 / 2} W_{c, i n, n}$. In the new variables, $W_{c, e q, 0}^{1 / 2}$ is proportional to $\exp \left(-y^{2} / 2\right)$. The actual counterpart of the operator $D[n ; s]$ in Subsection 2.2 is: $D[n ; s]=\left[s I+(n+1) \omega^{2} a^{+} D[n+\right.$ $1 ; s] a]^{-1}$. This $D[n ; s]$ can be evaluated in closed form [16], as we shall now outline. By using $a^{+}\left[s I+n \omega a^{+} a\right]^{-1}=\left[s I+n \omega\left(a^{+} a-1\right)\right]^{-1} a^{+}$, and after some iterative algebra, one finds that the dependence of $D[n ; s]$ on $a$ and on $a^{+}$occurs only through the product $a^{+} a$ and that, by rewriting $D[n ; s] \equiv D\left[n ; s ; a^{+} a\right]$, one gets:

$$
D\left[n ; s ; a^{+} a\right]=\left[s I+(n+1) \omega^{2} a^{+} a D\left[n+1 ; s ; a^{+} a-1\right]\right]^{-1}
$$

Let $f_{n^{\prime}}=H_{n^{\prime}}(y) \exp \left[-2^{-1} y^{2}\right], n^{\prime}=0,1,2, \ldots$ Then, the eigenfunctions of $a^{+} a$ and of $D\left[n ; s ; a^{+} a\right]$ are $f_{n^{\prime}}$. The eigenvalues of $a^{+} a$ are $n^{\prime}$ which, through iteration of Equation (23), yield directly those of $D\left[n ; s ; a^{+} a\right]$ as finite fractions, precisely due to the structure $a^{+} a-1$. In other words, $D[n ; s]$ (which, in principle, is an infinite continued fraction of operators) becomes, by iterating Equation (23), a finite fraction in $a^{+} a$. A posteriori, one confirms for the actual harmonic oscillator that the resulting $D[n ; s=\epsilon](\epsilon>0)$ is Hermitian and has nonnegative eigenvalues. See [16] for further details.

\section{Closed Classical Many-Particle Systems: Long-Time Approximation and Arrow of Time}

We shall present an outline of the main developments, omitting lengthy arguments and equations, which can be seen in [14]. We treat a closed large system of many $(N \gg 1)$ classical nonrelativistic particles, in $d$ spatial dimensions $(d=1,2,3)$, with spatial coordinates $\mathbf{x}_{1}, \ldots, \mathbf{x}_{N}(\equiv[x])$ and momenta $\mathbf{q}_{1}, \ldots, \mathbf{q}_{N}(\equiv[q])$. All particles, which are identical, have mass $m$. Let $x_{i, \alpha}$ and $q_{i, \alpha}$ be the Cartesian components of $\mathbf{x}_{i}$ and $\mathbf{q}_{i}$, respectively $(i=1, \ldots, N, \alpha=1, \ldots d)$. Neither a $h b$ nor external friction mechanisms nor external forces are assumed. The interaction potential is: $V=\sum_{i, j=1, i<j}^{N} V_{i, j}\left(\left|\mathbf{x}_{i}-\mathbf{x}_{j}\right|\right)$ and we suppose that all $V_{i, j}\left(\left|\mathbf{x}_{i}-\mathbf{x}_{j}\right|\right)$ are repulsive $(\geq 0)$ and tend quickly to zero for large $\left|\mathbf{x}_{i}-\mathbf{x}_{j}\right|$. The physical idea is that the very large number of degrees of freedom (which be at thermal equilibrium with one another) in the system play the role of a $h b$. The non-equilibrium classical distribution function is: $W_{c}=W_{c}([x],[q] ; t)$. Boltzmann's equilibrium (canonical) distribution at absolute temperature $T$ is : $W_{c, e q}=\exp \left[-\beta\left((2 m)^{-1} \Sigma_{i=1}^{N} \Sigma_{\alpha=1}^{d} q_{i, \alpha}^{2}+V\right)\right]$.

The initial non-equilibrium distribution $W_{c, i n}$, to be regarded as known, is quite arbitrary in practice. Instead of considering the most general initial state, we shall choose a class of $W_{c, i n}$ 's, which will be: (i) qualitatively consistent with the idea [8,22], typical of Information Theory, that one should employ only distribution functions compatible with the limited information available which, in turn, refers to expectation values not of all possible dynamical variables but only of an observable subset of such variables (these ideas being imposed for $t=0$ only, but not for $t>0$ ); (ii) also consistent with standard variables employed in Equilibrium Statistical Mechanics and Fluid Dynamics [2-5]. Then, we shall treat a class of an explicit ansätze for $W_{c, i n}$ which will depend on a finite number of functions (actually, $2+d$ ) of one single position vector, $\mathbf{x}: \lambda_{k}=\lambda_{k}(\mathbf{x}), k=0,2$, and $\lambda_{1, \alpha}=\lambda_{1, \alpha}(\mathbf{x}), \alpha=1, \ldots, d$ (all of them being independent on time and on momenta). The expression for $W_{c, i n}$ in terms of $\lambda_{k}$ and $\lambda_{1, \alpha}$ has 
appeared previously [3,5] (and is related to the Massieu-Planck function [5]). In short, $W_{c, i n}$ at $t=0$ will be chosen to describe thermal equilibrium with homogeneous temperature $T$ for large distances but non-equilibrium for intermediate and short distances (with spatial inhomogeneities). The ansatz is:

$$
\begin{aligned}
W_{c, i n}= & (N !)^{-1} \exp \left[-\int d^{d} \mathbf{x}\left(\lambda_{0}(\mathbf{x}) \sum_{i=1}^{N} \delta^{(d)}\left(\mathbf{x}_{i}-\mathbf{x}\right)+\sum_{\alpha=1}^{d} \lambda_{1, \alpha}(\mathbf{x}) \sum_{i=1}^{N} \frac{q_{i, \alpha}}{m}\right.\right. \\
& \left.\times \delta^{(d)}\left(\mathbf{x}_{i}-\mathbf{x}\right)+\lambda_{2}(\mathbf{x}) \sum_{i=1}^{N}\left(\frac{\mathbf{q}_{i}^{2}}{2 m}+\frac{1}{2} \sum_{j=1, j \neq i}^{N} V_{i, j}\left(\left|\mathbf{q}_{i}-\mathbf{q}_{j}\right|\right)\right) \delta^{(d)}\left(\mathbf{x}_{i}-\mathbf{x}\right)\right]
\end{aligned}
$$

Consistently with (i)-(ii) above, the $\lambda$ 's will be uniquely determined (through standard recipes in Information Theory) in terms of $2+d$ x-dependent observables (also independent on time and on momenta) typically employed in Fluid Dynamics, which, by assumption, are known at $t=0$ : mass density, fluid velocity and some suitable energy density [5,14]. For simplicity, no observables associated to angular momentum are included. How does the equilibrium temperature $T$ appear in this closed system, without $h b$ ? We assume that $W_{c, i n}$ describes thermal equilibrium at temperature $T$ at large $\mathbf{x}$ but off-equilibrium at finite $\mathbf{x}$. We accept that $\lambda_{2}(\mathbf{x})$ approaches quickly a non-vanishing constant, $\lambda_{2}(\infty)$, as $|\mathbf{x}|$ tends to $\infty$ along any direction and that the same holds for $\lambda_{0}(\mathbf{x})$. A similar statement holds for $\lambda_{1, \alpha}(\mathbf{x})$, the corresponding (large- $|\mathbf{x}|$ ) limiting value being zero. At finite $\mathbf{x}$, the off-equilibrium $\lambda_{2}(\mathbf{x})$, $\lambda_{0}(\mathbf{x})$ and $\lambda_{1, \alpha}(\mathbf{x})$ do depend on $\mathbf{x}$ and, so, differ from their respective constant (large- $\left.|\mathbf{x}|\right)$ limiting values, which describe equilibrium. Then, consistency is achieved ( $T$ being thereby introduced) if, in the thermodynamical limit, $\lambda_{2}(\infty)$ tends to $\left(k_{B} T\right)^{-1}$ (plus corrections which approach zero in that limit). The dominant contributions to various statistical averages at $t=0$ come from large $\mathbf{x}$, up to corrections which tend to vanish as $N$ increases. The very large number of degrees of freedom involved in the largest part of the system (for large $|\mathbf{x}|$ ) are at equilibrium at $T$. For consistency, the $2+d \mathbf{x}$-dependent dynamical variables known at $t=0$ (mass density, fluid velocity and some suitable energy density) have to fulfill the corresponding behaviour as $|\mathbf{x}|$ tends to $\infty$ along any direction. For details, see [14].

The reversible Liouville equation reads:

$$
\frac{\partial W_{c}}{\partial t}=\Sigma_{i=1}^{N} \Sigma_{\alpha=1}^{d}\left[\frac{\partial V}{\partial x_{i, \alpha}} \frac{\partial W_{c}}{\partial q_{i, \alpha}}-\frac{q_{i, \alpha}}{m} \frac{\partial W_{c}}{\partial x_{i, \alpha}}\right]
$$

Let $[n]$ denote a set of nonnegative integers $(n(i=1, \alpha=1), \ldots, n(i=N, \alpha=d))$ and let $n=\sum_{l=1}^{N} \Sigma_{\alpha=1}^{d} n(l, \alpha)$. Let $[d q]=\prod_{i=1}^{N} \prod_{\alpha=1}^{d} d q_{i, \alpha}$. We introduce non-equilibrium moments $W_{[n]}$ of $W$ (using products of Hermite polynomials, by generalizing Equation (3) with all integrations in $(-\infty,+\infty))$ :

$$
\int[d q] \prod_{i=1}^{N} \prod_{\alpha=1}^{d} \frac{H_{n(i, \alpha)}\left(q_{i, \alpha} /\left(2 m k_{B} T\right)^{1 / 2}\right)}{\left(\pi^{1 / 2} 2^{n(i, \alpha)} n(i, \alpha) !\right)^{1 / 2}} W_{c}([x],[q], t) \equiv W_{c}(x ;[n] ; t)=W_{c}([n])
$$

If $W_{c}=W_{c, e q}$, then $W_{c, e q}([0])([0]=(0,0, \ldots, 0)=(n(i=1, \alpha=1)=0, \ldots, n(i=N, \alpha=d)=$ $0)=[n=0])$ is proportional to $\exp [-\beta V]$ and $W_{c, e q}([n])=0,[n] \neq[0]$ (say, $\left.n \neq 0\right)$. Equation (26) can also be applied to $W_{c, i n}$ and gives the corresponding initial moments, $W_{c, i n}([n])$. We shall work with the 
symmetrized moments $g([n])=W_{c, e q}([0])^{-1 / 2} W_{c}([n])$. One gets an infinite reversible three-term linear recurrence for $g([n])$ 's, generalizing Equations (4) and (5). It reads:

$$
\begin{aligned}
& \frac{\partial g(n(1,1), \ldots, n(j, \beta), \ldots, n(N, d))}{\partial t} \\
= & -\Sigma_{l=1}^{N} \Sigma_{\alpha=1}^{d}\left[M_{l, \alpha ; n(l, \alpha) ;+} g(n(1,1), \ldots, n(l, \alpha)+1, \ldots, n(N, d))\right. \\
& \left.+M_{l, \alpha ; n(l, \alpha) ;-} g(n(1,1), \ldots, n(l, \alpha)-1, \ldots, n(N, d))\right] \\
M_{l, \alpha ; n(l, \alpha) ;+}= & {\left[\frac{(n(l, \alpha)+1) k_{B} T}{m}\right]^{1 / 2}\left[\frac{\partial}{\partial x_{l, \alpha}}-\frac{1}{2 k_{B} T} \frac{\partial V}{\partial x_{l, \alpha}}\right] } \\
M_{l, \alpha ; n(l, \alpha) ;-}= & {\left[\frac{n(l, \alpha) k_{B} T}{m}\right]^{1 / 2}\left[\frac{\partial}{\partial x_{l, \alpha}}+\frac{1}{2 k_{B} T} \frac{\partial V}{\partial x_{l, \alpha}}\right] }
\end{aligned}
$$

The Laplace transform of the hierarchy Equation (27) is the actual many-particle counterpart of Equation (6) with $\sigma^{-1}=0$. Such a Laplace transform (with $N \gg 1$ ) can be formally solved in terms of linear operators $D[[n] ; s]$, which generalize the previous $D[n ; s]$. For details, see [14]. All $D[[n] ; s]$ are square matrices, due to the indices $i=1, \ldots, N$ and $\alpha=1, \ldots, d$. In turn, each matrix element in those square matrices is an ordinary linear integral operator, arising from the partial differential operators $M_{l, \alpha ; n(l, \alpha) ;+}$ and $M_{l, \alpha ; n(l, \alpha) ;-}$, as $l, \alpha$ and $n(l, \alpha)$ vary. The $D[[n] ; s]$ fulfill the following formal hierarchy [which generalizes Equation (7)]:

$$
D[[n] ; s]=\left[s I-M_{+,[n+1]} D[[n+1] ; s] M_{-,[n]}\right]^{-1}
$$

The linear operators $M_{ \pm,[n]}$ are rectangular matrices, the elements of which are formed out of the partial differential operators $M_{l, \alpha ; n(l, \alpha) ;+}$ and $M_{l, \alpha ; n(l, \alpha) ;-} . M_{+,[n+1]}$ can be shown to be the adjoint of $-M_{-,[n]}$. By iterating Equation (30) indefinitely, one can express formally the linear operator $D[[n] ; s]$ as an operator continued fraction, which depends on all partial differential operators $M_{l, \alpha ; n(l, \alpha) ;+}$ and $M_{l, \alpha ; n(l, \alpha) ;-}$ and generalizes the operator continued fraction for $D[n ; s]$. See [14]

By generalizing the iterative arguments in Subsection 2.3, it follows that, for both $V \neq 0$ and $V=0$, $D[[n] ; \epsilon]$ for $\epsilon>0$ is a Hermitian operator with non-negative eigenvalues for $n \geq n_{0} \geq 1$ [14].

A few remarks for the case $V \equiv 0$ may be clarifying. In the Laplace transform of Equation (27), let us perform a spatial Fourier transformation from configuration space $\left(\mathbf{x}_{1}, \ldots, \mathbf{x}_{N}\right)$ to wavevector space $\left(\mathbf{k}_{1}, \ldots, \mathbf{k}_{N}\right) \equiv[k]$. Let $e(k ; N) \equiv k_{B} T \sum_{j=1}^{N}(2 m)^{-1} \mathbf{k}_{j}^{2}$. Then, the Fourier transform $D_{1}[[k] ;[n] ; s]$ of $D[[n] ; s]$ for $\operatorname{Re} s>0$ is an ordinary continued fraction, given in Equations (16)-(18) with $e(k)$ replaced by $e(k ; N)$. Then, with such a replacement, the properties of $D_{1}(k ; n ; s)$ given in Subsection 2.4 also hold for $D_{1}[[k] ;[n] ; s]$. Notice that $D_{1}[[k] ;[n] ; s=0]$ diverges as $e(k ; N)^{-1 / 2}$ if $e(k ; N) \rightarrow 0$. On the other hand, and contrary to what happened for one particle in one spatial dimension [recall the comment after Equation (21)], $\int[d k] D_{1}[[k] ;[n] ; s=0]$ converges near $e(k ; N)=0$. This would suggest that the actual counterpart of Equation (9) (containing $D[[n] ; \epsilon]$ acting on various $M_{l, \alpha ; n(l, \alpha) ;-} g(n(1,1), \ldots, n(l, \alpha)-$ $1, \ldots, n(N, d))^{\prime}$ 's), when integrated over $[k]$ 's to come back to $[x]$-space, would converge at small $[k]$ 's as $\epsilon \rightarrow 0$, for the actual $V \neq 0$. Whether such a property holds is an open question.

In spite of the very involved structure of Equation (27) (and of its Laplace transform), we argue that a simple long-time approximation can be performed in it, for $V_{i, j} \geq 0$ (and vanishing quickly at large distances) and very large $N$ (in the thermodynamical limit), which generalizes that in Subsection 2.3. 
This approximation consists in fixing $s=\epsilon>0$ ( $\epsilon$ being small) in the whole hierarchy of operators $D[[n] ; s]$, for any $n \geq n_{0}(>0)$ which, then, become Hermitian operators $D[[n] ; \epsilon]$ with no negative eigenvalues. It is crucial that $s$-dependences be kept in $D[[n] ; s]$, for $n<n_{0}$. Notice that the non-vanishing factors $n(l, \alpha)^{1 / 2}$ and $((n(l, \alpha)+1))^{1 / 2}$ in $M_{l, \alpha ; n(l, \alpha) ;-}$ and $M_{l, \alpha ; n(l, \alpha) ;+}$, respectively, tend to reduce, as the $n(l, \alpha)$ 's increase, the relative importance of having fixed $s=\epsilon$ and the contribution of the latter in the $D[[n] ; s=\epsilon]$ 's with $n \geq n_{0}$. This is a genuine feature of the $D[[n] ; s=\epsilon]$ 's. See [14], where it was seen that, by imposing $n_{0}>2$, the long-time approximation is exactly consistent with all hydrodynamical balance equations. For simplicity, we discard all the initial moments $W_{c, i n}([n])$ for $n \geq n_{0}$. We regard $D\left[\left[n_{0}\right] ; \epsilon\right]$ as a fixed (s-independent) operator, yielding all $g\left(\left[n_{0}\right]\right)$ in terms of all $g\left(\left[n_{0}-1\right]\right)$. The choice $\epsilon=0$ was made in [14]. By using [16] and [18,19], the arguments in [14] are easily seen to hold for $\epsilon>0$. All that leads to a closed approximate hierarchy for $g([n])$ 's (with initial moments $\left.W_{c, i n}([n])\right)$, with $n<n_{0}$, which appears to yield an approximate irreversible evolution towards thermal equilibrium at $T . g([n])$ 's and, then, $W_{c}([n])$ relax the quicker the larger $n$, provided that $n<n_{0} . W_{c}([0])$ would dominate the approach towards equilibrium for $t \rightarrow+\infty$. See [14]. All that appears to work based on the general properties of $D\left[\left[n_{0}\right] ; \epsilon\right]$ : for quantitative studies, some ansatz or approximation should be provided directly for it. An arrow of time would follow approximately, in the present case.

As an extreme example, let $n_{0}=1$ (which is strictly consistent only with the hydrodynamical balance equation for mass): see [14] for $n_{0}=2,3$. By making the above long-time approximation and taking inverse Laplace transforms, one finds directly the irreversible Smoluchowski-like equation for the $[n=0]$ moment, which generalizes Equation (13) $([n=0]$ meaning $n(1,1)=0, \ldots, n(j, \beta)=$ $0, \ldots, n(N, d)=0)$ :

$$
\begin{aligned}
\frac{\partial g([n=0])}{\partial t}= & \sum_{l=1}^{N} \Sigma_{\alpha=1}^{d} M_{l, \alpha ; n(l, \alpha)=0 ;+} \times \\
& \left(\sum_{l^{\prime}=1}^{N} \Sigma_{\alpha^{\prime}=1}^{d}[D[[n=1] ; \epsilon]]_{l, \alpha ; l^{\prime}, \alpha^{\prime}} M_{l^{\prime}, \alpha^{\prime} ; n\left(l^{\prime}, \alpha^{\prime}\right)=1 ;-}\right) g([n=0])
\end{aligned}
$$

The operator $D[[n=1] ; \epsilon]$ (Hermitian, with non-negative eigenvalues) has, as a square matrix, the matrix elements $[D[[n=1] ; \epsilon]]_{l, \alpha ; l^{\prime}, \alpha^{\prime}}$. The initial condition is $W_{c, e q}([0])^{-1 / 2} W_{c, i n}([0])$. Compare with [14].

\section{Open Quantum-Mechanical One-Dimensional System without Dissipation: General Aspects}

We shall consider a quantum Brownian particle $q B p$ of mass $m(>0)$ and momentum operator $-i \hbar(\partial / \partial x)$, in one spatial dimension $x$, with (Hermitian) quantum Hamiltonian:

$$
H=-\frac{\hbar^{2}}{2 m} \frac{\partial^{2}}{\partial x^{2}}+V
$$

with a real potential $V=V(x) \geq 0$ vanishing quickly for $|x| \rightarrow+\infty$. $\hbar$ is Planck's constant. All eigenvalues $E_{j}$ of $H$ sweep the continuous positive real half-line: $0 \leq E_{j}<+\infty$. Such a continuous spectrum has, typically, a double degeneracy, associated to different asympotic conditions at $x \rightarrow \pm \infty$, with the same energy. The continuous variable $j(-\infty<j<+\infty)$ labels all states and distinguishes degenerate states. Thus, if $\varphi_{j}(x)$ is an eigenfunction of $H: H \varphi_{j}(x)=E_{j} \varphi_{j}(x)$.

The example considered in Subsection 2.1 (namely, a Brownian particle moving in air at room temperature) could be invoked here as well: the particle should now be described by Quantum 
Mechanics. This Section presents a purely formal generalization of Subsections 2.1 and 2.2 for the non-equilibrium statistical evolution of a $q B p$ subject to $V(x)$, and in the presence of a $h b$ at thermal equilibrium at $T$, in the idealized case of vanishing dissipation. The time evolution for $t>0$ of the $q B p$ is given by the density operator $\rho=\rho(t)$ (a statistical mixture of quantum states), with the initial condition $\rho(t=0)=\rho_{i n}$. For $t \geq 0, \rho(t)$ is a Hermitian and positive-definite linear operator acting in a Hilbert space. Unless otherwise stated, we shall not impose that $\rho(t)$ be normalized. The time evolution of the $q B p$ in differential form is described by the operator equation $([H, \rho]=H \rho-\rho H)$ :

$$
\frac{\partial \rho}{\partial t}=\frac{1}{i \hbar}[H, \rho]
$$

We consider the matrix element $\langle x-y|\rho(t)| x+y\rangle$ of $\rho(t)$ in generic eigenstates, $|x-y\rangle,|x+y\rangle$, of the quantum position operator. The quantum Wigner function $W=W(x, q ; t)$, determined by $\rho$, is $[6,7]$ :

$$
W(x, q ; t)=\frac{1}{\pi \hbar} \int_{-\infty}^{+\infty} d y \exp \left[\frac{i 2 q y}{\hbar}\right]\langle x-y|\rho(t)| x+y\rangle
$$

The initial non-equilibrium Wigner function at $t=0$ is $W_{i n}$, given by Equation (34) if $\rho=\rho_{i n}$. For $t>0$, the exact dissipationless quantum master equation (QME) for $W[6,7]$ is:

$$
\begin{aligned}
\frac{\partial W(x, q ; t)}{\partial t}= & -\frac{q}{m} \frac{\partial W(x, q ; t)}{\partial x}+M_{Q} W \\
M_{Q} W= & \int_{-\infty}^{+\infty} d q^{\prime} W\left(x, q+q^{\prime} ; t\right) \int_{-\infty}^{+\infty} \frac{i d y}{\pi \hbar^{2}}[V(x+y)-V(x-y)] \\
& \times \exp \left[-\frac{i 2 q^{\prime} y}{\hbar}\right]=\frac{d V}{d x} \frac{\partial W}{\partial q}-\frac{\hbar^{2}}{3 ! 2^{2}} \frac{d^{3} V}{d x^{3}} \frac{\partial^{3} W}{\partial q^{3}}+\cdots
\end{aligned}
$$

As $\hbar \rightarrow 0$, Equation (35) becomes formally, by dropping all $\hbar$-dependent terms (containing $\partial^{n} W / \partial p^{n}$ in Equation (36), $\left.n=3,5, \ldots\right)$, the classical Liouville equation Equation (2), with $W \rightarrow W_{c}$ [6,7]. We shall assume that, as $|q| \rightarrow+\infty, W(x, q ; t) \rightarrow 0$ quickly, for fixed $x$ and long $t$. Then, $\int_{-\infty}^{+\infty} d q W(x, q ; t) q^{n}$ converges, for any integer $n \geq 0$. Equation (35) readily implies that $(\partial / \partial t) \int_{-\infty}^{+\infty} d x \int_{-\infty}^{+\infty} d q W(x, q ; t)=0$.

A stationary Wigner function is any $t$-independent solution of Equation (35). We are not interested on arbitrary stationary Wigner functions, but only on a very specific one (denoted by $W_{e q}$ ), namely, that which accounts for the thermal equilibrium state of the $q B p$, at temperature $T=\left(k_{B} \beta\right)^{-1}$, with the $h b$. Like in the classical case, the solutions of Equations (35) and (36) are not expected to approach $W_{e q}$ exactly, unless some approximation be made. $W_{e q}$ arises from the canonical ( $t$-independent) density operator $\rho_{e q}=\exp [-\beta H]$, with matrix elements:

$$
\left\langle x-y\left|\rho_{e q}\right| x+y\right\rangle=\sum_{j} \exp \left[-\beta E_{j}\right] \varphi_{j}(x-y) \varphi_{j}(x+y)^{*}
$$

$\sum_{j}$ is a short-hand notation denoting integration over the whole continuous spectrum of $j\left(\int_{-\infty}^{+\infty} d j\right)$. In turn, $\rho_{e q}$ determines $W_{e q}(x, p)$, through Equation (34):

$$
\begin{aligned}
& W_{e q}(x, q)=\frac{1}{\pi \hbar} \int_{-\infty}^{+\infty} d y \exp \left[\frac{i 2 q y}{\hbar}\right] \sum_{j} \exp \left[-\beta E_{j}\right] \varphi_{j}(x-y) \varphi_{j}(x+y)^{*} \\
& -\frac{q}{m} \frac{\partial W_{e q}}{\partial x}+M_{Q} W_{e q}=0, \frac{\partial W_{e q}}{\partial t}=0
\end{aligned}
$$


We remark that if one has constructed some general stationary Wigner function, by solving Equation (39), further additional information should be fed in, so as to select uniquely that solution of Equation (39) which yields precisely $W_{e q}$. Such a situation will be met in Section 5. Some known genuine difficulties of the quantum case are that $W_{e q}(x, p)$ is neither Gaussian in $q$ nor known in closed form, for a general $V$ [6,7]. In Subsection 4.1 we shall start the generalizations of Subsections 2.1 and 2.2 to the actual quantum situation, so as to search for ways for solving or, at least, bypassing the genuine difficulties, mentioned above, of the quantum case. An alternative representation for $W_{e q}$ has been given by Wigner [6]: we shall review it shortly in Subsection 4.2 and use it in Section 5.

\subsection{Orthogonal Polynomials $H_{Q, n}^{\prime}$ Generated by $W_{e q}$ in Equation (38), Moments and Hierarchy}

We shall introduce the denumerably infinite family of all (unnormalized) polynomials in $q$, $H_{Q, n}^{\prime}=H_{Q, n}^{\prime}(q)(n=0,1,2,3, \ldots)$, orthogonalized in $q$ (for fixed $x$ ) by using the equilibrium Wigner function $W_{e q}$ Equation (38) as weight function. Let $q_{0}$ be some fixed (scaling) momentum: $q_{0}$ could equal $q_{e q}$ [Equation (3)], but not necessarily. By choosing $H_{Q, 0}^{\prime}(q)=1$, for $n \neq n^{\prime}$ and any $x$ (left unintegrated), we impose:

$$
\int_{-\infty}^{+\infty} \frac{d q}{q_{0}} W_{e q}(x, q) H_{Q, n}^{\prime}(q) H_{Q, n^{\prime}}(q)=0
$$

The $H_{Q, n}^{\prime}$ 's, depending parametrically on $x$ for $n \geq 1$, generalize the standard Hermite polynomials, and will be used for the time evolution. We shall look for the $H_{Q, n}^{\prime}(q)$ 's as $\left(y_{0}=q / q_{0}\right)$ :

$$
H_{Q, n}^{\prime}(q)=y_{0}^{n}+\sum_{j=1}^{n} \epsilon_{n, n-j} y_{0}^{n-j}
$$

$\epsilon_{n, n-j}$ being $q$-independent (but $x$-dependent, in general): see Appendix A. The orthonormalized polynomials are $H_{Q, n}^{\prime}(q) /\left(h_{n}^{\prime}\right)^{1 / 2}$, with the (x-dependent) normalization factor:

$$
h_{n}^{\prime} \equiv \int_{-\infty}^{+\infty} \frac{d q}{q_{0}} W_{e q}(x, q) H_{Q, n}^{\prime}(q)^{2}
$$

The lowest normalization factors $h_{0}^{\prime}$ and $h_{1}^{\prime}$ are given in Appendix A.

We shall treat general situations in which the $q B p$ could be out of thermal equilibrium with the $h b$ at $t=0$ (and, hence, for $t>0$ ). We shall analyze them by using Equation (35), for the non-equilibrium $W$. The study will also enable to discuss, as a consistency check, the case in which the qBp be at thermal equilibrium with the $h b$ at any $t$. The $H_{Q, n}^{\prime}(q)$ 's suggest the following new moments $W_{n}(n=0,1,2, \ldots)$ :

$$
W_{n}=W_{n}(x ; t)=\int_{-\infty}^{+\infty} \frac{d q}{q_{0}} H_{Q, n}^{\prime}(q) W(x, q ; t)
$$

The initial condition $W_{i n, n}$ for $W_{n}$ is obtained by replacing $W$ by the initial non-equilibrium Wigner function $W_{\text {in }}$ in Equation (43). One has the following (formal) expansion for $W$ :

$$
W=W_{e q}(x, q) \sum_{n=0}^{+\infty} W_{n}(x ; t) \frac{H_{Q, n}^{\prime}(q)}{h_{n}^{\prime}}
$$


For $W=W_{e q}(x, q)$, Equation (43) yields $W_{e q, n}=0$ if $n>0$, and $W_{e q, 0}=h_{0}$.

For the actual dissipationless case, the role of $H_{Q, n}^{\prime}(q)$, of the $W_{n}$ 's and of Equation (44) can be appreciated through the following formal argument, which extends to the quantum case behaviours met in the classical case (Subsections 2.1-2.3). One could argue that, at least for some initial non-equilibrium conditions and after some approximations, $W_{n}(x ; t)$ would approach or be related to $W_{e q, n}$. Then, for long-time and approximately, the dominant moment would be $W_{0}(x ; t)$, while any $W_{n}(x ; t)$ with $n>0$ would be negligible, $W_{n}$ being the smaller, the larger $n$ and $t(>0)$. One has: $\int_{-\infty}^{+\infty} d x \int_{-\infty}^{+\infty} d\left(q / q_{0}\right) W(x, q ; t)=\int_{-\infty}^{+\infty} d x W_{0}(x ; t)$. The above argument, even if it would not seem essentially wrong, is recognizedly loose for vanishing dissipation, unless some approximation be made. It will be revisited in Section 6, with dissipation.

The transformation of Equations (35) and (36) into a linear hierarchy for the new moments $W_{n}$ is outlined in Appendix A. The hierarchy in Equations (74), (75) and so on is exact and very general, but it requires to know the eigenfunctions $\varphi_{j}(x)$ and the eigenvalues $E_{j}$. Our limited aim here was to show that orthogonal polynomials and a non-equilibrium hierarchy exist formally for Equation (35). Then, we shall neither delve further into this nor treat the issue of long-time approximations for Equations (74), (75) and so on. In Sections 5 and 6, we shall treat other alternatives, with repulsive quadratic plus quartic potentials.

\subsection{The Wigner Representation for $W_{\text {eq }}$ for High Temperature, Near the Classical Limit}

We remind the following representation for $W_{e q}$ [given in Equation (38)] as a series in the standard Hermite polynomials $H_{n}\left(q / q_{e q}\right)[6]$ :

$$
W_{e q}=W_{c, e q} \sum_{n=0}^{+\infty} a_{0, n} H_{n}\left(\frac{q}{q_{e q}}\right)
$$

$W_{c, e q}$ is Boltzmann's classical distribution function, given in Subsection 2.1, and $q_{e q}$ was given in Equation (3). The $a_{0, n}=a_{0, n}(x)$ 's are coefficients, which depend on $\hbar, V(x)$ and $\beta$, with $a_{0, n} \equiv 0$ for $n=1,3,5, \ldots$ The expansion Equation (45) holds for high temperature and near the classical limit (small $\beta$ and $\hbar$ ). The $a_{0, n}$ 's, $n=0,2,4,6,8, \ldots$, are non-vanishing in general: those for $n=0,2,4$ have been given in [6]. Here, it will suffice to quote only $a_{0,0}$, through the first terms in a series expansion into powers of $\hbar)$ :

$$
\begin{aligned}
a_{0,0}(x)=1 & +\frac{\hbar^{2}}{8}\left[\frac{\beta^{3}}{3 m}\left(\frac{d V}{d x}\right)^{2}-\frac{2 \beta^{2}}{3 m} \frac{d^{2} V}{d x^{2}}\right]+\frac{\hbar^{4} \beta^{2}}{64 m^{2}}\left[-\frac{4 \beta}{15} \frac{d^{4} V}{d x^{4}}+\frac{\beta^{4}}{18}\left(\frac{d V}{d x}\right)^{4}\right. \\
& \left.-\frac{22 \beta^{3}}{45}\left(\frac{d V}{d x}\right)^{2} \frac{d^{2} V}{d x^{2}}+\frac{8 \beta^{2}}{15} \frac{d V}{d x} \frac{d^{3} V}{d x^{3}}+\frac{2 \beta^{2}}{5}\left(\frac{d^{2} V}{d x^{2}}\right)^{2}\right]+\cdots
\end{aligned}
$$

All terms in Equation (46) contribute if $\hbar \neq 0$ and $\beta \neq 0$, even if $V=m \omega^{2} x^{2} / 2\left(a_{0,0}(x) \neq 1\right.$, then). The key information, which does select uniquely the equilibrium Wigner function $W_{e q}$, out of the set of all stationary Wigner functions, is encoded in the factor $W_{c, e q}$ and in the fact that $W_{e q} \rightarrow W_{c, e q}$ for any $V$, if $\hbar \rightarrow 0$ and small $\beta$. This is, of course, consistent with Equation (46) and with $a_{0,0}(x) \rightarrow 1$ in the classical high-temperature limit for any $V$. As shown in [6], all the remaining non-vanishing $a_{0, n}(x)$ 's, $n=2,4,6,8, \ldots$, are determined recursively in terms of $a_{0,0}(x)$. 


\section{Quantum-Mechanical One-Dimensional Model with Quadratic Plus Quartic V: No Dissipation}

In this Section, we shall restrict to a quadratic plus quartic $V(\geq 0)$ :

$$
V=V(x)=\frac{m \omega^{2} x^{2}}{2}+V_{1}, V_{1}=V_{1}(x)=\frac{g x^{4}}{4 !}
$$

that is, we deal with a quantum anharmonic oscillator, with harmonic frequency $\omega(>0) . g$, real and $\geq 0$, is an anharmonicity parameter. Then, $H$ is defined in a denumerably infinite Hilbert space. $H$ has a denumerably infinite discrete spectrum of real eigenvalues $E_{j}(\geq 0), j=1, \ldots$ and there is no continuous spectrum. For the actual quadratic plus quartic $V(x)$, the (formal) series expansion of the integral operator $M_{Q}$ into powers of $\hbar^{2}$ reduces exactly to the first two terms shown in Equation (36). In this Section, we shall also bypass the genuine difficulties of the quantum case: the representation of $W_{e q}$ and the construction of the $H_{Q, n}^{\prime}$ 's will now differ considerably from those in Section 4.

\subsection{Alternative Series for $W_{e q}(x, q)$, Orthogonal Polynomials Generated by It and Hierarchy}

We shall deal with $W_{e q}(x, q)$ (as given in Equation (38) in general, and in Equation (45) for small $\hbar$ and $\beta$ ) for the special case in Equation (47). We shall recast $W_{e q}(x, q)$ into a new series in the standard Hermite polynomials, which should be not restricted to the regime of small $\hbar$ and $\beta$ [as is Equation (45)]. For that purpose, we shall introduce:

$$
\begin{aligned}
\alpha & =\frac{\hbar \omega[1+\cosh (\beta \hbar \omega)]}{2 \sinh (\beta \hbar \omega)}, q_{Q, e q}=+[2 m \alpha]^{1 / 2} \\
W_{e q}^{(0)} & =W_{e q}^{(0)}(x, q)=\exp \left[-\frac{1}{\alpha}\left(\frac{q^{2}}{2 m}+\frac{m \omega^{2} x^{2}}{2}\right)\right]
\end{aligned}
$$

Notice that $q_{Q, e q} \neq q_{e q}$ (although $q_{Q, e q} \rightarrow q_{e q}$ for $\hbar \omega \rightarrow 0$ ). One looks for $W_{e q}(x, q)$ as the new formal series in the standard Hermite polynomials $H_{n}\left(q / q_{Q, e q}\right)$ :

$$
W_{e q}=W_{e q}^{(0)} \sum_{n=0}^{+\infty} a_{e q, n} H_{n}\left(\frac{q}{q_{Q, e q}}\right)
$$

with new coefficients $a_{e q, n}=a_{e q, n}(x)$, depending on $\hbar, m, \omega, \beta$ and $x$. We emphasize that Equations (45) and (50) are different expansions. Based upon the well known $\rho_{e q}$ for $g=0$ [7,23-25], a direct computation shows that $W_{e q}^{(0)}$ is the exact Wigner function for the quantum harmonic oscillator for any $m, \omega, \beta$ and $\hbar$. Then, if $g=0$, it follows that $a_{e q, n}=0$ for $n=1,2,3,4, \ldots$ and that $a_{e q, 0}$ is $x$-independent. This justifies the interest of Equation (50): it can be expected to converge, at least, for small positive $g$ for finite $\beta$.

Let us characterize $a_{e q, 0}$ first. As $\int_{-\infty}^{+\infty}\left(d q / q_{Q, e q}\right) W_{e q}=\left(q_{e q} / q_{Q, e q}\right) \int_{-\infty}^{+\infty}\left(d q / q_{e q}\right) W_{e q}$ and by using Equations (45) and (50), one gets:

$$
a_{e q, 0}=\frac{q_{e q}}{q_{Q, e q}} a_{0,0}(x) \exp \left[+\frac{1}{\alpha} \frac{m \omega^{2} x^{2}}{2}\right] \exp [-\beta V]
$$

If $g=0$ (for finite $\beta$ and $\hbar$ ), the $x$-dependences of $a_{0,0}(x)$, $\exp \left[+(2 \alpha)^{-1} m \omega^{2} x^{2}\right]$ and $\exp [-\beta V]$ cancel out in Equation (51) exactly, so that $a_{e q, 0}$ is $x$-independent. For the new series Equation (50), the 
additional information which does select uniquely $W_{e q}$, out of the set of all stationary Wigner functions, is encoded in the factor $W_{e q}^{(0)}$ and in $a_{e q, 0}(x)$ [determined, through Equation (51), in terms of $a_{0,0}$ given, in turn, in Equation (46)]. Equations (39), (50), (47) and (49) yield a three-term linear recurrence relation for the $a_{e q, n}$ 's: see Appendix B.

By generalizing Equation (50), one could expand the non-equilibrium Wigner function in terms of the $H_{n}\left(q / q_{Q, e q}\right)$ 's, in terms of non-equilibrium moments $a_{n}$ 's. For large $t(>0)$ and, at least, for some suitable class of initial non-equilibrium conditions, one could argue whether the $a_{n}$ 's would approach approximately or be related, in some sense, to the $a_{e q, n}$ 's which, as shown above, are non-vanishing. Then, for long-time, all $a_{n}, n=2,4,6, \ldots$ would be non-vanishing as well and should be taken into account, which would make their analysis rather difficult. The above argument, which is rather loose in the absence of dissipation, will be revisited in Section 6 with friction included. Anyway, the above argument will provide motivation for the following developments.

We shall come back to the denumerably infinite family of polynomials $H_{Q, n}^{\prime}(q)(n=0,1,2,3, \ldots)$, orthogonalized in $q$ (for fixed $x$ ) by using $W_{e q}$ [Equation (38), in the form of Equation (50)] as weight function. Here, we shall use Equation (40), with $q_{0}$ replaced by $q_{Q, e q}$. For the actual $V$, given in Equation (47), we shall recast those $H_{Q, n}^{\prime}(q) \equiv H_{Q, n}(y)\left(y=q / q_{Q, e q}\right)$ into another form. Strictly speaking, the $H_{Q, n}^{\prime}(q)$ 's considered in Equation (40) for a general $V$ coincide with the $H_{Q, n}(y)$ 's to be analyzed here only when Equation (47) holds. By assumption, we search for $H_{Q, n}(y)$ to equal the standard Hermite polynomial $H_{n}(y)$ plus a remainder. The latter is another polynomial in $y$ of degree smaller than $n$. We shall write:

$$
H_{Q, n}(y)=H_{n}(y)+\sum_{j=1}^{n} \sigma_{n, n-j} H_{n-j}(y)
$$

with $n=1,2,3, \ldots$ and ( $y$-independent) coefficients $\sigma_{n, n-j}$ (given in Appendix B), which depend on $x$ and $\beta$ (these two dependences not being explicited). The orthonormalized polynomials are $H_{Q, n}\left(q / q_{Q, e q}\right) / h_{Q, n}^{1 / 2}$, with the (x-dependent) normalization factor:

$$
h_{Q, n} \equiv \int_{-\infty}^{+\infty} \frac{d q}{q_{Q, e q}} W_{e q}(x, q) H_{Q, n}\left(q / q_{Q, e q}\right)^{2}
$$

See Appendix B. The alternative hierarchies off-equilibrium and at equilibrium following from $H_{Q, n}^{\prime}(q)=H_{Q, n}\left(q / q_{Q, e q}\right)$ and $h_{n}^{\prime}=h_{Q, n}\left(\right.$ with $q_{0}$ replaced by $\left.q_{Q, e q}\right)$ are outlined in Appendix B.

\section{Quantum-Mechanical One-Dimensional Model with Quadratic Plus Quartic $V$ : Dissipation}

\subsection{First Model: Equilibrium Wigner Function Dependent on Dissipation}

Here, we shall suppose that the evolution of the $q B p$, in the presence of the $h b$, is also subject to non-vanishing friction. We shall regard the $q B p$ as an open quantum system, also described by Equations (32) and (47) in the same denumerably infinite Hilbert space. Recall that open quantum systems constitute a very active research field, which is common to several modern branches of quantum physics (quantum optics, laser theory, atoms and electromagnetic radiation in cavities, spin relaxation 
dynamics, decoherence, ion traps, quantum information,... ) [23-31]. For $t>0$, we shall assume that the QME for $W$, including dissipation, is:

$$
\begin{aligned}
\frac{\partial W(x, q ; t)}{\partial t} & =-\frac{q}{m} \frac{\partial W(x, q ; t)}{\partial x}+M_{Q} W+M_{D} W \\
M_{D} W & =\left[\gamma+\frac{\gamma}{2}\left(x \frac{\partial}{\partial x}+q \frac{\partial}{\partial q}\right)+\gamma \frac{\alpha}{2 m \omega^{2}}\left(\frac{\partial^{2}}{\partial x^{2}}+m^{2} \omega^{2} \frac{\partial^{2}}{\partial q^{2}}\right)\right] W
\end{aligned}
$$

with the same $M_{Q}$ given in Equation (36). The linear operator $M_{D}$, with the same $\alpha$ as in Equation (48), accounts for the dissipation on the $q B p$ due to the $h b$. The key real parameter $\gamma(\geq 0)$ accounts for friction effects. Equations (54) and (55) readily imply that $(\partial / \partial t) \int_{-\infty}^{+\infty} d x \int_{-\infty}^{+\infty} d q W(x, q ; t)=0$. Equations (54) and (55) are: (a) the standard equations describing a single mode of the electromagnetic field inside a laser cavity [23,24], if $g=0$; (b) a toy model for the inclusion of weak nonlinearities in (a) (say, due to a nonlinear medium within the cavity), along the lines pursued in [25], if $g \neq 0$.

First, we shall look for the physically acceptable equilibrium solution, denoted as $W_{e q, \gamma}(x, q)$, of Equation (54) $\left(\partial W_{e q, \gamma}(x, q) / \partial t=0\right)$. An important property is that, provided that $g=0, W_{e q}^{(0)}$, given in Equation (49) [with the same $q_{Q, e q}$ as in Equation (48)], is the equilibrium solution of Equation (54) (with $\gamma \neq 0)$ as a direct computation shows. Then, if $g=0, W_{e q, \gamma}(x, q)=W_{e q}^{(0)}$, which is $\gamma$-independent. However, for $g \neq 0$, a qualitatively new feature is that $W_{e q, \gamma}(x, q)$ is indeed $\gamma$-dependent and, hence, it is not given by the right-hand-sides of either Equation (34) (with $\rho=\exp (-\beta H)$ ) or Equation (50). This dependence of quantum equilibrium distributions in certain models, like the one in Equations (54) and (55), on the dissipation mechanism is one of the additional difficulties met in the quantum case, to be treated in this Subsection. We remind that, in order to avoid physically that difficulty, an interesting generalization of Equation (1) to the quantum case (to order $\hbar^{2}$ ), in the regime of small $\hbar$ and $\beta$, has been carried out in [32].

Then, by reminding Equation (50) for the frictionless case, we shall look for $W_{e q, \gamma}(x, q)$ if $\gamma \neq 0$ and $g \neq 0$ as the new series using the standard Hermite polynomials:

$$
W_{e q, \gamma}=W_{e q}^{(0)} \sum_{n=0}^{+\infty} a_{e q, \gamma, n} H_{n}\left(\frac{q}{q_{Q, e q}}\right)
$$

with new ( $\gamma$-dependent) coefficients) $a_{e q, \gamma, n}\left(\neq a_{e q, n}\right)$ : see Appendix C. In order to select uniquely $W_{e q, \gamma}$ in the actual case with dissipation, we need a choice for $a_{e q, \gamma, 0}$. We shall choose $a_{e q, \gamma, 0}=a_{e q, 0}$, as given in Equation (51) ( $\gamma$-independent). Such a choice for $a_{e q, \gamma, 0}$ seems a natural one (not leading to inconsistencies) although, recognizedly, it also seems less compelling than that for $a_{e q, 0}$ in Equation (51) (which was necessary, for consistency). Then, all $a_{e q, \gamma, n}(x)$ 's, $n=1,2,3,4, \ldots$, are determined recurrently in terms of $a_{e q, \gamma, 0}(x)$, through Equation (89), through the obvious generalizations of Equations (77) and (78). Notice that $a_{e q, \gamma, n}(x) \neq 0$ for odd $n^{\prime}\left(n^{\prime}=1,3,5, \ldots\right)$. For $g=0$, with nonvanishing $\beta$, $\hbar$ and $\gamma, a_{e q, \gamma, n}(x)=0$ for $n=1,2,3,4, \ldots$ while $a_{e q, \gamma, 0}(x)$ is $\mathrm{x}$-independent, consistently.

Like in the dissipationless case, we shall introduce the new denumerably infinite family of all polynomials in $y=q / q_{Q, e q}, H_{Q, \gamma, n}=H_{Q, \gamma, n}(y)(n=0,1,2,3, \ldots)$, orthogonalized in $q$ by using the equilibrium Wigner function $W_{e q, \gamma}$ given in Equation (56) as weight function. Then:

$$
\int_{-\infty}^{+\infty} \frac{d q}{q_{Q, e q}} W_{e q, \gamma}(x, q) H_{Q, \gamma, n}(y) H_{Q, \gamma, n^{\prime}}(y)=0, n \neq n^{\prime}
$$


We impose that $H_{Q, 0}(y)=1$ and that for $n \geq 1, H_{Q, \gamma, n}(y)$ equals the standard Hermite polynomial $H_{n}(y)$ plus another polynomial in $y$ of degree smaller than $n$. Then:

$$
H_{Q, \gamma, n}(y)=H_{n}(y)+\sum_{j=1}^{n} \sigma_{\gamma, n, n-j} H_{n-j}(y)
$$

with $n=1,2,3, \ldots$ The coefficients $\sigma_{\gamma, n, n-j}$ depend on $x, \beta$ and $\gamma$ : they are determined recurrently through Equation (57). See Appendix C. We omit the normalizing factors of $H_{Q, \gamma, n}(y)$.

Using the $H_{Q, \gamma, n}(y)$ 's, we introduce the new non-equilibrium moments $W_{\gamma, n}(n=0,1,2, \ldots)$ of $W$ :

$$
W_{\gamma, n}=W_{\gamma, n}(x ; t)=\int_{-\infty}^{+\infty} \frac{d q}{q_{Q, e q}} H_{Q, \gamma, n}(y) W(x, q ; t)
$$

Notice that $\int_{-\infty}^{+\infty} d x \int_{-\infty}^{+\infty} d\left(q / q_{Q, e q}\right) W(x, q ; t)=\int_{-\infty}^{+\infty} d x W_{\gamma, 0}(x ; t)$. At equilibrium, the moments of $W_{e q, \gamma}$ determined by Equation (59) are: $W_{e q, \gamma, n}=0$ for $n=1,2,3,4, \ldots$ while $W_{e q, \gamma, 0}=$ $\pi^{1 / 2} \exp \left[-\frac{1}{\alpha} \frac{m \omega^{2} x^{2}}{2}\right] a_{e q, \gamma, 0}$. Then, one can proceed to the non-equilibrium recurrence relation for the $W_{\gamma, n}$. It will suffice to give the lowest two equations in the recurrence in Appendix C.

\subsection{Second Model: Equilibrium Wigner Function Independent on Dissipation (Lindblad's Theory)}

A characteristic feature of Equations (54) and (55) was that the corresponding equilibrium Wigner function did depend on dissipation. Here, we shall treat another model for the evolution of the $q B p$, in the presence of the $h b$, also with the choice Equation (47) for $V(x)$ and subject to non-vanishing dissipation, based upon $[25,29,33,34]$ and to all orders in $\hbar$ : then, the equilibrium Wigner function will be independent on dissipation. For an economical description, it will be convenient not to limit to the Wigner function $W$, but to employ also an equivalent operator formulation in terms of the density operator $\rho(t)$, which is uniquely determined by $W$. In fact, any matrix element of $\rho(t)$ is given, by performing an inverse Fourier transform of Equation (34), by:

$$
\langle x-y|\rho(t)| x+y\rangle=\int_{-\infty}^{+\infty} d q \exp \left[\frac{-i 2 q y}{\hbar}\right] W(x, q ; t)
$$

Let $a, a^{+}$be the standard destruction and annihilation operators for the quantum harmonic oscillator: $a=2^{-1 / 2}\left[\alpha_{0} x+\alpha_{0}^{-1}(\partial / \partial x)\right]$ and $a^{+}=2^{-1 / 2}\left[\alpha_{0} x-\alpha_{0}^{-1}(\partial / \partial x)\right]\left(\alpha_{0}=(m \omega / \hbar)^{1 / 2}\right)$. The superscript + will always denote the adjoint. Then, with Equation (47), one has: $H=H_{0}+V_{1}$, with $H_{0}=\hbar \omega_{0}\left(a^{+} a+1 / 2\right)$ and $V_{1}=g_{1}\left(a+a^{+}\right)^{4}\left(g_{1}=g /\left[2^{2} 4 ! \alpha_{0}^{4}\right]\right)$.

We shall suppose that the actual time evolution of the $q B p$, including dissipation, is a quantum Markov process, described by the following QME:

$$
\frac{\partial \rho}{\partial t}=\frac{1}{i \hbar}[H, \rho]+D \rho
$$

$D$ ("the dissipator"), accounting for the dissipation on the $q B p$ due to the $h b$, is a linear operator. In order to characterize $D$, we shall introduce first the following linear ( $t$-independent) operators $A\left(\omega^{\prime}\right)$ and $A^{+}\left(\omega^{\prime}\right)$ as:

$$
\begin{aligned}
{\left[H, A\left(\omega^{\prime}\right)\right] } & =-\hbar \omega^{\prime} A\left(\omega^{\prime}\right) \\
{\left[H, A^{+}\left(\omega^{\prime}\right)\right] } & =\hbar \omega^{\prime} A^{+}\left(\omega^{\prime}\right) \\
A^{+}\left(\omega^{\prime}\right) & =A\left(-\omega^{\prime}\right)
\end{aligned}
$$


$D, A\left(\omega^{\prime}\right)$ and $A^{+}\left(\omega^{\prime}\right)$ act on the actual denumerably infinite Hilbert space. $\omega^{\prime}$ (with dimension $(\text { time })^{-1}$ ) is a real number, as shown in general [29]. $A\left(\omega^{\prime}\right)$ and $A^{+}\left(\omega^{\prime}\right)$ are named the eigen-operators of $H[33,34]$ and also Lindblad's operators. They are supposed to be dimensionless. $A^{+}\left(\omega^{\prime}\right)$ is a traceless operator [29]. Equations (62) and (63) imply: $\left[H, A^{+}\left(\omega^{\prime}\right) A\left(\omega^{\prime}\right)\right]=0$. Equation (64) implies that $A\left(\omega^{\prime}\right)$ is Hermitian only if $\omega^{\prime}=0$ : then, $\left[A\left(\omega^{\prime}=0\right), H\right]=0$. For the time being, we shall not deal with $A\left(\omega^{\prime}\right)$, $A^{+}\left(\omega^{\prime}\right)$ and the associated $\omega^{\prime}$ for $g_{1} \neq 0$ : we shall turn to that pending task in Appendix $\mathrm{D}$, for the sake of completeness.

We shall make more precise the model in Equation (61) by assuming that $D \rho$ has the structure [33,34]:

$$
D \rho=\sum_{\omega^{\prime \prime}} \gamma\left(\omega^{\prime \prime}\right)\left[\left[A\left(\omega^{\prime \prime}\right) \rho, A^{+}\left(\omega^{\prime \prime}\right)\right]+\left[A\left(\omega^{\prime \prime}\right), \rho A^{+}\left(\omega^{\prime \prime}\right)\right]\right]
$$

The summation in Equation (65) is performed only over two real values: $\omega^{\prime \prime}=\omega^{\prime}$ and $\omega^{\prime \prime}=-\omega^{\prime}$. $\gamma\left(\omega^{\prime \prime}\right)$ are real and positive numbers: they have dimension $(\text { time })^{-1}$ and, physically, they play the role of relaxation rates. Technically, Equations (61)-(65) (known as Lindblad's formulation for Markovian open quantum systems) give the most general Markovian and time-homogeneous master equation describing a non-unitary evolution of $\rho$ that is trace preserving and completely positive for any $\rho_{i n}$. See [25,29,31] for presentations of several alternative arguments leading to Equation (65). For $g \neq 0$ (say, $g_{1} \neq 0$ ), the representation of Equation (61) [with $D \rho$ given in Equation (65)] by means of the Wigner function is different from Equation (54) [with $M_{D}$ given in Equation (55)]. It is crucial to notice that the canonical ( $t$-independent) density operator $\rho_{e q}(=\exp (-\beta H))$ with vanishing friction as considered in Subsection 4.1 (with $\left[\rho_{e q}, H\right]=0$ ) fulfills:

$$
\begin{aligned}
\rho_{e q} A\left(\omega^{\prime}\right) & =\exp \left[\beta \hbar \omega^{\prime}\right] A\left(\omega^{\prime}\right) \rho_{e q} \\
\rho_{e q} A^{+}\left(\omega^{\prime}\right) & =\exp \left[-\beta \hbar \omega^{\prime}\right] A^{+}\left(\omega^{\prime}\right) \rho_{e q}
\end{aligned}
$$

We shall not treat the class of models in Equations (61) and (65) in its full generality. Rather, by inspiring ourselves on $[25,29]$, we shall add the assumption:

$$
\gamma\left(-\omega^{\prime}\right)=\exp \left[-\beta \hbar \omega^{\prime}\right] \gamma\left(\omega^{\prime}\right)
$$

Equations (61), (65) and (68) fully characterize the quantum model with dissipation on which we shall concentrate in this Subsection. Then, Equations (65), (66), (67) and (68) imply the crucial property:

$$
D \rho_{e q}=0
$$

See [29] for the proof of Equation (69). As $\partial \rho_{e q} / \partial t=0, \rho_{e q}$ is, indeed, an equilibrium state of the QME Equation (61) describing the $q B p$ in the presence of the $h b$, with dissipation included through Equations (65) and (68). Then, the equilibrium Wigner function determined by $\rho_{e q}$ coincides exactly with $W_{e q}$ in Equation (50). Thus, the orthogonal polynomials for the actual Lindblad's theory coincide with the $H_{Q, n}\left(q / q_{Q, e q}\right)$ 's studied in Subsection 5.1. The non-equilibrium moments $W_{Q, n}$ for the actual Lindblad's theory are also given in Equations (43) and (44), with $H_{Q, n}^{\prime}(q)=H_{Q, n}\left(q / q_{Q, e q}\right), h_{n}^{\prime}=h_{Q, n}$ and with $q_{0}$ replaced by $q_{Q, e q}$.

A very important example of $A\left(\omega^{\prime}\right)$ and the above formulation is provided by the QME for a quantized radiation mode with frequency $\omega$ in a laser cavity, with $V_{1}=0$, when dissipation is included [25,28-30]. 
Then, with $g=0\left(g_{1}=0\right)$, the representation of Equation (61) and of $D \rho$ [through Equations (65) and (68)] by means of the Wigner function can be shown to coincide with Equations (54) and (55).

At this stage, we shall focus shortly on our main target, namely, the interest of the $W_{Q, n}$ 's (and, hence, of the $H_{Q, n}$ 's) for the time evolution, in the present framework with friction. Like in Subsection 5.1, we multiply Equation (61), with $D \rho$ given in Equations (65) and (68), by $H_{Q, n}\left(q / q_{Q, e q}\right) / q_{Q, e q}$ and integrate over $q$. That yields: $\partial W_{Q, n}(x, t) / \partial t=\Lambda_{n, 1}(x, t)+\Lambda_{n, 2}(x, t)$, in which we manipulate, so as to express the resulting equation solely in terms of the $W_{Q, n}$ 's. $\Lambda_{n, 1}(x, t)$ is the contribution from $\frac{1}{i \hbar}[H, \rho]$, which has been treated in Appendix B. $\Lambda_{n, 2}(x, t)$ is the contribution from $D \rho$, given in Equation (65), which gives rise to new features. In order to deal with $\Lambda_{n, 2}(x, t)$, we use, successively, the formal closure relation $I=\int d z|z\rangle\langle z|$, Equations (60) and (44), with $H_{Q, n}^{\prime}(q), h_{n}^{\prime}$ and $q_{0}$ replaced by $H_{Q, n}\left(q / q_{Q, e q}\right), h_{Q, n}$ and $q_{Q, e q}$, respectively. As expected, the net result is: $\Lambda_{n, 2}(x, t)=$ $\sum_{n^{\prime}=0}^{+\infty} \int d x^{\prime} \lambda_{n, 2 ; n^{\prime}}\left(x, x^{\prime}\right) W_{Q, n^{\prime}}\left(x^{\prime} ; t\right)$, with some kernel $\lambda_{n, 2 ; n^{\prime}}\left(x, x^{\prime}\right)$, determined by $A\left(\omega^{\prime}\right), A^{+}\left(\omega^{\prime}\right)$ and the $H_{Q, n}\left(q / q_{Q, e q}\right)$ 's and $H_{Q, n^{\prime}}\left(q / q_{Q, e q}\right)$ 's. We shall omit the complicated $\lambda_{n, 2 ; n^{\prime}}\left(x, x^{\prime}\right)$, because it will be unnecessary for the discussion that follows.

First, let us consider the case in which the $q B p$ is at thermal equilibrium, so that $W=W_{e q}$ in Equation (50). All equilibrium moments $W_{e q, n^{\prime}}=0$ for $n^{\prime}=1,2,3,4, \ldots$ and $W_{e q, n^{\prime}=0}=h_{Q, 0}$ fulfill, for any $n=0,1,2,3, \ldots$, not only $\Lambda_{n, 1}=0$ but, by virtue of Equation (69), $\Lambda_{n, 2}=0$ as well.

Based upon the above equilibrium case, we consider next the time evolution for large $t$. It may be adequate to address here the discussion in Subsection 4.1, after Equation (44). One would argue that $W(x, q ; t)-W_{e q}(x, q)$ should be the smaller (and eventually tend to zero), the larger $t$ is (at least, for a suitable, possibly restricted, class of initial non-equilibrium conditions). Then, to argue that, for long-time, the dominant moment would be $W_{Q, 0}(x ; t)$, while any $W_{Q, n}(x ; t)$ with $n>0$ would be negligible ( $W_{Q, n}$ being the smaller, the larger $n$ and $t(>0)$ ) would not seem essentially incorrect. This (even in the lack of a rigorous control) would suggest the following approximation scheme, for suitably large $t$ and some restricted set of initial non-equilibrium conditions. In the hierarchy $\partial W_{Q, n}(x, t) / \partial t=$ $\Lambda_{n, 1}(x, t)+\Lambda_{n, 2}(x, t)$, let us restrict to the equations for $n=0$ and $n=1$ for suitably large $t$, and we neglect in them all $W_{Q, n^{\prime}}(x, t)$ 's with $n^{\prime} \geq 2$. In the resulting equation for $n=1$, we also neglect $\partial W_{Q, 1}(x, t) / \partial t$, obtain formally $W_{Q, 1}$ in terms of $W_{Q, 0}$ and reshuffle the last expression into the equation for $\partial W_{Q, 0}(x, t) / \partial t=\Lambda_{0,1}(x, t)+\Lambda_{0,2}(x, t)$. This would provide an approximate evolution equation for $W_{Q, 0}(x, t)$ alone, for large $t$, describing the approach to thermal equilibrium of the $q B p$, with dissipation.

\section{Conclusions, Discussions and Open Problems}

This work contains two parts, very closely related to each other, summarized below with more detail. The first part (a review of previous work, including certain improved analysis) has dealt with classical systems: Sections 2 and 3. It is not warranted that the main issues of the latter (the long-time approximation and so on) could be extended to general quantum systems. As an attempt towards that, the second part has been devoted to one-dimensional quantum systems: Sections 4, 5 and 6, with new material dealing with more difficult problems and, hence, having a formal and limited scope.

(1) We have extended previous work by other authors on open classical one-dimensional systems off-equilibrium, with dissipation. In the present work, we have considered those systems, both with or without dissipation, as well as closed classical many-particle ones. In all cases, equilibrium 
(Boltzmann's) classical canonical distribution functions, which are Gaussian in momenta, have been used as weight functions to generate families of orthogonal polynomials in momenta (namely, the standard Hermite polynomials), for fixed spatial coordinates. Distinguishing and simplifying features of all classical systems considered here are that dependences on classical momenta and those on spatial coordinates factorize (as no magnetic fields are considered), so that those orthogonal polynomials in momenta are independent on positions. Three-term linear hierarchies for the non-equilibrium classical moments (which depend only on the spatial coordinates) have been studied and solved formally, in terms of certain operator continued fractions. For an open classical one-dimensional system with dissipation, the equilibrium distribution is independent on the latter: the analysis (with repulsive potentials which vanish at large distance) shows that, for long times, the lowest moment dominates the evolution towards thermal equilibrium with the $h b$, while higher order moments are subdominant. The latter study has been extended, under certain long-time approximation, to the case without dissipation, which does provide valuable hints for generalizations to non-equilibrium closed classical interacting nonrelativistic many-particle systems, with repulsive potentials vanishing at large distance. Then, the very large number of degrees of freedom of the system plays the role of a $h b$, and the initial states are assumed to correspond to thermal equilibrium at large distances (thereby introducing the equilibrium temperature, $T$ ), but to non-equilibrium situations at finite distances. The canonical equilibrium distribution leads to orthogonal polynomials which are suitable products of Hermite polynomials and leads to generalize the non-equilibrium moment method. We emphasize that for closed classical three-dimensional many-particle interacting systems, the non-equilibrium hierarchy based upon moments treated here is radically different from the well known one due to Born, Bogoliubov, Green, Kirkwood and Yvon (BBGKY) [2,3] for classical distribution functions, and that the operator continued fractions appear to yield, in a long-time approximation, irreversibility and thermalization of the whole system at $T$, for long times (and, hence, an approximate arrow of time): then, the lowest moment also dominates the evolution towards thermal equilibrium. The conclusion also appears to hold for a non-equilibrium classical closed plasma, with the degrees of the classical electromagnetic field included [15]. The classical open one-dimensional case corresponding to a harmonic oscillator (with a repulsive potential which grows at large distance), with vanishing dissipation, has been discussed very shortly, for completeness. The analysis of non-equilibrium for classical systems, considered here, has been based on continued fractions of certain linear operators. Some crucial properties of those operator continued fractions (Hermiticity, absence of negative eigenvalues) have been inferred through the analysis of examples and iterative arguments. Moreover, those operators have been constructed in compact forms, in outline, for free particles and for a classical harmonic oscillator. We emphasize that such operators appear to be very interesting objects, which would deserve much more mathematical attention than the limited one devoted to them here.

(2) Non-equilibrium quantum interacting systems present various conceptually new difficulties. We have concentrated on descriptions through Wigner functions. We quote some quantum difficulties of the latter, on which we have focused: canonical equilibrium Wigner distributions $W_{e q}$ are not Gaussian in momenta, their dependences on the latter and on spatial coordinates do not factorize and they may depend on the dissipation mechanism (for various open systems). Due to such difficulties, we have limited ourselves to open quantum one-dimensional interacting models, so as to investigate various procedures 
to construct the $W_{e q}$ 's, and the very construction of families of orthogonal polynomials in momenta which have those $W_{e q}$ 's as weight functions, to all orders in Planck's constant. We have considered, first, a general repulsive potential $V(x)$ (vanishing suitably for $|x| \rightarrow+\infty$ ) without dissipation due to the $h b$ first and, later, we have turned to a repulsive quadratic plus quartic potential (either without or with dissipation). We have studied the $W_{e q}$ 's, in all those cases. For a repulsive quadratic plus quartic potential with dissipation, we have considered two models, inspired on laser theory: (i) one, in which $W_{e q}$ does depend on dissipation; (ii) another one, using Lindblad's theory, in which $W_{e q}$ is independent on dissipation. In all those cases, we have outlined the construction of the new families of orthogonal polynomials in momenta, which have the corresponding $W_{e q}$ as weight function. We have employed those new families of orthogonal polynomials (depending parametrically also on spatial coordinates), in order to construct new moments of the non-equilibrium Wigner function. Hierarchies for the non-equilibrium moments (which depend only on spatial coordinates) have been treated in outline. Then, it seems possible to generalize the developments of the open classical case for the open quantum one, at least formally. The construction of stationary or equilibrium solutions of Equation (61) in a general case (in which Equations (68) and (69) do NOT hold) would proceed, if the eigen-operators are known, through a procedure similar to that in Subsection 6.1. See [31] for theorems and constructive methods to find stationary solutions of Equation (61) in general cases.

The issues of carrying through some limited control of convergence and of long-time approximations in the open quantum one-dimensional case (generalizing the ones for the classical cases in Sections 2 and 3) are more difficult and have not been addressed here. We have also omitted generalizations to quantum closed interacting many-particle systems (and, hence, issues related to quantum indistinguishability). Then, many questions remain open in the approach to non-equilibrium quantum systems through orthogonal polynomials, moment methods and long-time approximations, compared to classical cases.

\section{Acknowledgements}

The author is grateful to Craig Callender for inviting him to contribute to the Special Issue Arrow of Time of Entropy. The author acknowledges the financial support of Project FIS2008-01323, Ministerio de Ciencia e Innovacion, Spain. He is an associate member of BIFI (Instituto de Biocomputacion y Fisica de los Sistemas Complejos), Universidad de Zaragoza, Zaragoza, Spain. He thanks A. Rivas for discussions and facilities.

\section{References}

1. Wallace, D. Reading list for the philosophy of statistical mechanics. Available online: http:// users.ox.ac.uk/ mert0130/papers/smreading.doc (accessed on 13 February 2012).

2. Kreuzer, H.J. Nonequilibrium Thermodynamics and Its Statistical Foundations; Clarendon Press: Oxford, UK, 1981.

3. Balescu, R. Equilibrium and Nonequilibrium Statistical Mechanics; John Wiley and Sons: New York, NY, USA, 1975.

4. Liboff, R.L. Kinetic Theory, 2nd ed.; John Wiley (Interscience): New York, NY, USA, 1998. 
5. Zubarev, D.; Morozov, V.G.; Röpke, G. Statistical Mechanics of Nonequilibrium Processes; Akademie Verlag: Berlin, Germany, 1996; Volume I.

6. Wigner, E.P. On the quantum correction for thermodynamic equilibvrium. Phys. Rev. 1932, 40, 749-759.

7. Hillery, M.; O'Connell, R.F.; Scully, M.O.; Wigner, E.P. Distribution functions in physics: Fundamentals. Phys. Rep. 1984, 106, 121-167.

8. Penrose, O. Foundations of statistical mechanics. Rep. Prog. Phys. 1979, 42, 1937-2006.

9. Brinkman, H.C. Brownian motion in a field of force and the diffusion theory of chemical reactions. Physica 1956, 22, 29-34.

10. Risken, H. The Fokker-Planck Equation, 2nd ed.; Springer: Berlin, Heidelberg, Germany, 1989.

11. Coffey, W.T.; Kalmykov, Yu. P. ; Waldron, J.T. The Langevin Equation, 2nd ed.; World Scientific: Singapore, 2004.

12. Coffey, W.T.; Kalmykov, Yu. P.; Titov, S.V.; Mulligan, B.P. Wigner function approach to the quantum Bronian motion of a particle in a potential. Phys. Chem. Chem. Phys. 2007, 9, 3361-3382.

13. Hochstrasser, U.W. Orthogonal polynomials. In Handbook of Mathematical Functions; Abramowitz, M., Stegun, I.A., Eds.; Dover: New York, NY, USA, 1965.

14. Alvarez-Estrada, R.F. New hierarchy for the Liouville equation, irreversibility and Fokker-Planck-like structures. Ann. Phys. (Leipzig) 2002, 11, 357-385.

15. Alvarez-Estrada, R.F. Liouville and Fokker-Planck dynamics for classical plasmas and radiation. Ann. Phys. (Leipzig) 2006, 15, 379-415.

16. Alvarez-Estrada, R.F. Nonequilibrium quasi-classical effective meson gas: Thermalization. Eur. Phys. J. A 2007, 31, 761-765.

17. Alvarez-Estrada, R.F. Nonequilibrium quantum anharmonic oscillator and scalar field: High temperature approximations. Ann. Phys. (Berlin) 2009, 18, 391-409.

18. Alvarez-Estrada, R.F. Brownian motion, quantum corrections and a generalization of the Hermite polynomials. J. Comput. Appl. Math. 2010, 233, 1453-1461.

19. Alvarez-Estrada, R.F. Classical systems: Moments, continued fractions, long-time approximations and irreversibility. AIP Conf. Proc. 2011, 1332, 261-262.

20. Alvarez-Estrada, R.F. Quantum Brownian motion and generalizations of the Hermite polynomials. J. Comput. Appl. Math. 2011, 236, 7-18.

21. Gautschi, W. Error functions and Fresnel integrals. In Handbook of Mathematical Functions; Abramowitz, M., Stegun, I.A., Eds.; Dover, New York, NY, USA, 1965.

22. Penrose, O.; Coveney, P.V. Is there a "canonical" non-equilibrium ensemble? Proc. R. Soc. Lond. 1994, A447, 631-646.

23. Louisell, W.H. Quantum Statistical Properties of Radiation; John Wiley and Sons: New York, NY, USA, 1973.

24. Haken, H. Laser Theory; Encyclopedia of Physics, Volume XXV/2c, Light and Matter Ic; Springer: Berlin, Heidelberg, Germany, 1970.

25. Gardiner, C.W.; Zoller, P. Quantum Noise, 3rd ed.; Springer: Berlin, Heidelberg, Germany, 2004.

26. Weiss, U. Quantum Dissipative Systems, 3rd ed.; World Scientific: Singapore, 2008. 
27. Joos, E.; Zeh, H.D.; Kiefer, C.; Giulini, D.; Kupsch, J.; Stamatescu, I.-O. Decoherence and the Appearance of a Classical World in Quantum Theory, 2nd ed.; Springer: Berlin, Heidelberg, Germany, 2003.

28. van Kampen, N.G. Stochastic Processes in Physics and Chemistry; Elsevier: Amsterdam, The Netherlands, 2001.

29. Breuer, H.-P.; Petruccione, F. The Theory of Open Quantum Systems; Oxford University Press: Oxford, UK, 2006.

30. Haroche, S.; Raimond, J.-M. Exploring the Quantum; Oxford University Press: Oxford, UK, 2008.

31. Rivas, A.; Huelga, S.F. Open Quantum Systems. An Introduction; Springer: Berlin, Heidelberg, Germany, 2011.

32. Coffey, W.T.; Kalmykov, Yu.P.; Titov, S.V.; Mulligan, B.P. Semiclassical Klein-Kramers and Smoluchowski equations for the Brownian motion of a particle in an external potential. J. Phys. A Math. Theor. 2007, 40, F91-F98.

33. Lindblad, G. On the generators of quantum dynamical semigroups Commun. Math. Phys. 1976, 48, 119-130.

34. Gorini, V.; Kossakowski, A.; Sudarshan, E.C.G. Completely positive semigroups of N-level systems. J. Math. Phys. 1976, 17, 821-825.

35. Garcia-Palacios, J.L.; Zueco, D. The Caldeira-Leggett quantum master equation in Wigner phase space: Continued-fraction solutions and applications to Brownian motion in periodic potentials. J. Phys. A Math. Gen. 2004, 37, 10735-10770. 


\section{Appendices}

\section{A. $\epsilon_{n, n-j}$ 's and Hierarchy at Equilibrium and Off-Equilibrium for Equations (35) and (36)}

We shall treat the low-order cases $n=1,2$, by using Equations (38), (40) and (41). After some algebra, one finds:

$$
\begin{aligned}
\epsilon_{1,0}=- & \frac{i \hbar}{2 q_{0}} \frac{\sum_{j} \exp \left[-\beta E_{j}\right]\left[\frac{d\left(\varphi_{j}(x-y) \varphi_{j}(x+y)^{*}\right)}{d y}\right]_{y=0}}{\left\langle x\left|\rho_{e q}\right| x\right\rangle} \\
\epsilon_{2,0}\left\langle x\left|\rho_{e q}\right| x\right\rangle+ & \frac{i \hbar \epsilon_{2,1}}{2 q_{0}} \sum_{j} \exp \left[-\beta E_{j}\right]\left[\frac{d\left(\varphi_{j}(x-y) \varphi_{j}(x+y)^{*}\right)}{d y}\right]_{y=0} \\
& -\frac{\hbar^{2}}{4 q_{0}^{2}} \sum_{j} \exp \left[-\beta E_{j}\right]\left[\frac{d^{2}\left(\varphi_{j}(x-y) \varphi_{j}(x+y)^{*}\right)}{d y^{2}}\right]_{y=0}=0 \\
\epsilon_{2,1}\left[\epsilon_{1,0} \frac{i \hbar}{2 q_{0}} \sum_{j}\right. & \exp \left[-\beta E_{j}\right]\left[\frac{d\left(\varphi_{j}(x-y) \varphi_{j}(x+y)^{*}\right)}{d y}\right]_{y=0} \\
& \left.-\frac{\hbar^{2}}{4 q_{0}^{2}} \sum_{j} \exp \left[-\beta E_{j}\right] \times\left[\frac{d^{2}\left(\varphi_{j}(x-y) \varphi_{j}(x+y)^{*}\right)}{d y^{2}}\right]_{y=0}\right] \\
= & \epsilon_{1,0} \frac{\hbar^{2}}{4 q_{0}^{2}} \sum_{j} \exp \left[-\beta E_{j}\right]\left[\frac{d^{2}\left(\varphi_{j}(x-y) \varphi_{j}(x+y)^{*}\right)}{d y^{2}}\right]_{y=0} \\
& -\frac{i \hbar^{3}}{8 q_{0}^{3}} \sum_{j} \exp \left[-\beta E_{j}\right]\left[\frac{d^{3}\left(\varphi_{j}(x-y) \varphi_{j}(x+y)^{*}\right)}{d y^{3}}\right]_{y=0}
\end{aligned}
$$

$\epsilon_{2,0}$ and $\epsilon_{2,1}$ are obtained by solving Equations (71) and (72). The lowest normalization factors are:

$$
h_{0}^{\prime}=\frac{\left\langle x\left|\rho_{e q}\right| x\right\rangle}{q_{0}}, h_{1}^{\prime}=\frac{\left\langle x\left|\rho_{e q}\right| x\right\rangle}{q_{0}}\left[\epsilon_{1,0} \epsilon_{2,1}-\epsilon_{2,0}-\epsilon_{1,0}^{2}\right]
$$

We shall transform Equations (35) and (36) into a linear hierarchy for the new moments $W_{n}$. For that purpose, we multiply Equation (35) by $H_{Q, n}^{\prime}(q) / q_{0}$, integrate over $q$ and operate, so as to express the resulting equation solely in terms of the $W_{n^{\prime}}$ 's. We carry out cancellations and simplifications, by using Equations (35) and (36) and the properties of the $H_{Q, n}^{\prime}(q)$ 's, which do play a crucial role. The computations become increasingly complicated as $n$ increases. It will suffice to display the first two equations in the hierarchy:

$$
\begin{aligned}
\frac{\partial W_{0}}{\partial t}= & -\frac{q_{0}}{m} \frac{\partial W_{1}}{\partial x}+\frac{q_{0}}{m} \frac{\partial\left(\epsilon_{1,0} W_{0}\right)}{\partial x} \\
\frac{\partial W_{1}}{\partial t}= & -\frac{q_{o}}{m} \frac{\partial W_{2}}{\partial x}+\frac{q_{0}}{m} \frac{\partial}{\partial x}\left[\left(\epsilon_{1,0}\left(\epsilon_{1,0}-\epsilon_{2,1}\right)+\epsilon_{2,0}\right) W_{0}\right]-\frac{q_{0}}{2 m} \frac{\partial \epsilon_{1,0}^{2}}{\partial x} W_{0} \\
& -\frac{q_{0}}{m} \frac{\partial}{\partial x}\left[\left(\epsilon_{1,0}-\epsilon_{2,1}\right) W_{1}\right]+\frac{q_{0}}{m} \frac{\partial \epsilon_{1,0}}{\partial x} W_{1}-\frac{1}{q_{0}} \frac{d V}{d x} W_{0}
\end{aligned}
$$

with initial condition $W_{i n, n}$. The following interesting check of consistency arises. One would expect that, at thermal equilibrium, the set formed by all equilibrium moments $\left(W_{e q, n}=0\right.$ if $n>0$, and $W_{e q, 0}=h_{0}$ ) fulfills the hierarchy in Equations (74), (75) and so on. However, it is not obvious, a priori, whether the above hierarchy can be automatically satisfied by those $t$-independent $W_{e q, n}$ 's, and, 
hence, whether some intricate cancellations need to be established a posteriori in order to display that consistency check. Direct computations show that $W_{e q, 1}=0$ and $W_{e q, 0}=h_{0}$ (with $\left.\partial W_{e q, 0} / \partial t=0\right)$ do fulfill Equation (74). In turn, Equation (75) is indeed satisfied by $W_{e q, 1}=W_{e q, 2}=0$ and $W_{e q, 0}=h_{0}$ in general, the corresponding proof being rather lengthy: we shall outline a check of it in Appendix B, when Equation (47) holds. .

\section{B. $W_{e q}$, Orthogonal Polynomials Generated by It and Hierarchy, When Equation (47) Holds}

Equations (39), (50), (47) and (49) yield the following three-term linear recurrence relation for the $a_{e q, n}$ 's [with $a_{e q, 0}$ given in Equation (51)]:

$$
\begin{aligned}
& -\frac{q_{Q, e q} 2(n+1)}{2 m}\left[\frac{d a_{e q, n+1}}{d x}-\frac{m \omega^{2} x}{\alpha} a_{e q, n+1}\right] \\
& -\frac{q_{Q, e q}}{2 m}\left[\frac{d a_{e q, n-1}}{d x}+\frac{1}{\alpha} \frac{d V_{1}}{d x} a_{e q, n-1}\right]+\frac{\hbar^{2}}{3 ! 2^{2}}\left[\frac{q_{Q, e q}}{2 m \alpha}\right]^{3} \frac{d^{3} V_{1}}{d x^{3}} a_{e q, n-3}=0
\end{aligned}
$$

with $a_{e q,-1}=a_{e q,-2}=a_{e q,-3}=0$. For even values of $n=0,2,4,6, \ldots$, the solutions for non-vanishing $a_{e q, 1}, a_{e q, 3}, a_{e q, 5}, \ldots$ are not acceptable. To understand this statement, take $n=0$. Then, Equation (76) yields $a_{e q, 1}=a_{e q, 1,0} \exp \left[+\frac{1}{\alpha} \frac{m \omega^{2} x^{2}}{2}\right], a_{e q, 1,0}$ being constant. $a_{e q, 1}$ diverges exponentially as $|x| \rightarrow+\infty$ and makes $\int d x W_{e q}$ to diverge. Then, we choose $a_{e q, 1,0}=0$. From that and through a similar analysis of the recurrence Equation (76) for $n=2,4,6, \ldots$, one concludes that $a_{e q, 1}=a_{e q, 3}=a_{e q, 5}=\cdots=0$. All the remaining $a_{e q, n}(x)^{\prime}$ s, $n=2,4,6, \ldots$ are to be determined recurrently below in terms of $a_{e q, 0}(x)$, through Equation (76) for odd values $n=1,3,5,7, \ldots$, once we impose that they (like $a_{e q, 1}$ above) do not diverge exponentially as $|x| \rightarrow+\infty$. With $a_{e q, 0} \neq 0$, one finds successively:

$$
\begin{aligned}
a_{e q, 2}(x)=\exp [ & \left.+\frac{1}{\alpha} \frac{m \omega^{2} x^{2}}{2}\right] \int_{-\infty}^{x} d x^{\prime} \exp \left[-\frac{1}{\alpha} \frac{m \omega^{2} x^{\prime 2}}{2}\right] \frac{(-1)}{4}\left[\frac{d a_{e q, 0}}{d x^{\prime}}+\frac{1}{\alpha} \frac{d V_{1}}{d x^{\prime}} a_{e q, 0}\right] \\
a_{e q, 4}(x)=\exp [ & \left.+\frac{1}{\alpha} \frac{m \omega^{2} x^{2}}{2}\right] \int_{-\infty}^{x} d x^{\prime} \exp \left[-\frac{1}{\alpha} \frac{m \omega^{2} x^{\prime 2}}{2}\right] \frac{(-1)}{8}\left[\left[\frac{d a_{e q, 2}}{d x^{\prime}}+\frac{1}{\alpha} \frac{d V_{1}}{d x^{\prime}} a_{e q, 2}\right]\right. \\
& \left.+\frac{\hbar^{2}}{3 ! 2^{2} 2 m \alpha^{2}} \frac{d^{3} V_{1}}{d x^{\prime 3}} a_{e q, 0}\right]
\end{aligned}
$$

and so on for $a_{e q, n}, n=6,8, \ldots$ We emphasize that $a_{e q, n} \neq a_{0, n}$ in Equation (45), and that $a_{e q, n} \neq 0$, for $n=2,4,6, \ldots$ For $g=0$, since $a_{e q, 0}$ is $x$-independent, one has $a_{e q, n}=0$, for $n=2,4,6, \ldots$ The new series Equation (50) is a nontrivial reordering (for finite $\beta$ and $\hbar$ ), of the Wigner series Equation (45). As $\hbar \rightarrow 0$ for small $\beta$, for finite $g$, the consistency of Equation (50) with Equation (45) and that of Equation (51) implies that $a_{e q, 0}(x) \rightarrow \exp \left[-\beta V_{1}\right]$ and $a_{e q, n}(x) \rightarrow 0$ for $n=2,4, \ldots$ The latter are consistent with Equations (77), (78) and so on. A posteriori, one sees that one could not have imposed $a_{e q, 0}(x)=1$ when $g \neq 0$.

The $\sigma_{n, n-j}$ 's in Equation (52) can be determined successively in terms of the $a_{e q, n}$ 's, by using Equations (40), (50), (52) and the orthogonality properties of the standard $H_{n}(y)$ 's. The computations become increasingly complex, as $n$ grows. One has, trivially, that $\sigma_{n, n-j}=0$, unless $j$ is even. We shall limit ourselves to give the lowest non-vanishing $\sigma_{n, n-j}$ :

$$
\begin{aligned}
& \sigma_{2,0}=-\frac{2^{3} a_{e q, 2}}{a_{e q, 0}}, \sigma_{3,1}=-\frac{2^{3} 4 ! a_{e q, 4}+2^{2} 3 ! a_{e q, 2}}{a_{e q, 0}+2^{2} a_{e q, 2}} \\
& \sigma_{4,0} a_{e q, 0}+2^{3}{ }_{-} 4,2 a_{e q, 2}+2^{4} 4 ! a_{e q, 4}=0, \sigma_{4,0}-\sigma_{4,2} \sigma_{2,0}-2 \sigma_{3,1}+\sigma_{2,0}\left(6+\sigma_{3,1}\right)=0
\end{aligned}
$$


Consistently with general properties [13], the $H_{Q, n}$ 's fulfill the recurrence relation:

$$
2 y H_{Q, n}(y)=H_{Q, n+1}(y)+C_{n} H_{Q, n-1}(y)
$$

for $n=0,1,2, \ldots$, with $C_{0} \equiv 0 . C_{n}$ are $y$-independent (but depend on $\beta$ and $x$ ). By using Equation (52) and the explicit expressions for the standard $H_{n}(y)$ 's, one can obtain the $C_{n}$ 's successively. We shall limit ourselves to give the lowest non-vanishing $C_{n}$ 's. One gets:

$$
C_{1}=2-\sigma_{2,0}, C_{2}=4+\sigma_{2,0}-\sigma_{3,1}
$$

The lowest ( $x$-dependent) normalization factors are:

$$
\begin{aligned}
& h_{Q, 0}=\pi^{1 / 2} \exp \left[-\frac{1}{\alpha} \frac{m \omega^{2} x^{2}}{2}\right] a_{e q, 0} . \\
& h_{Q, 1}=\pi^{1 / 2} \exp \left[-\frac{1}{\alpha} \frac{m \omega^{2} x^{2}}{2}\right] 2\left[a_{e q, 0}+4 a_{e q, 2}\right] \\
& h_{Q, 2}=\pi^{1 / 2} \exp \left[-\frac{1}{\alpha} \frac{m \omega^{2} x^{2}}{2}\right]\left[\left(8+\sigma_{2,0}^{2}\right) a_{e q, 0}+2^{3}\left(8+\sigma_{2,0}\right) a_{e q, 2}+2^{4} 4 ! a_{e q, 4}\right]
\end{aligned}
$$

We shall come back to the general situation in which the $q B p$ is off-equilibrium with the $h b$ for $t \geq 0$. We shall analyze it by using Equation (35) for the non-equilibrium $W$, now assuming Equation (47) and using Equations (43) and (44), with $q_{0}$ replaced by $q_{Q, e q}$ and with $H_{Q, n}^{\prime}(q)=H_{Q, n}\left(q / q_{Q, e q}\right), h_{n}^{\prime}=h_{Q, n}$. With the latter replacements, Equation (43) defines new moments $W_{Q, n}$ (and $W_{i n, Q, n}$, for $W=W_{i n}$ ).

We shall transform Equations (35) and (36) into another linear hierarchy for the $W_{Q, n}$ 's. For that purpose, we multiply Equation (35) by $H_{Q, n}\left(q / q_{Q, e q}\right) / q_{Q, e q}$, integrate over $q$ and operate, so as to express the resulting equation solely in terms of the $W_{Q, n^{\prime}}$ 's. Again, the issue of performing a check of consistency arises, which will be carried out at a later stage.

We have performed cancellations and simplifications, by using the structure of Equations (35) and (36) and of the properties of the $H_{Q, n}\left(q / q_{Q, e q}\right)$ 's, which do play a crucial role. The computations become increasingly complicated as $n$ increases. In particular, Equations (76) for $n=1$ and Equation (79) play a crucial role. We get the following three-term recurrence relation:

$$
\begin{aligned}
& \frac{\partial W_{Q, 0}}{\partial t}=-\frac{q_{Q, e q}}{2 m} \frac{\partial W_{Q, 1}}{\partial x} \\
& \frac{\partial W_{Q, 1}}{\partial t}=-\frac{q_{Q, e q}}{2 m} \frac{\partial W_{Q, 2}}{\partial x}+\frac{q_{Q, e q}}{2 m} \frac{\partial}{\partial x}\left[\sigma_{2,0} W_{Q, 0}\right]-\frac{q_{Q, e q}}{2 m} 2 \frac{\partial W_{Q, 0}}{\partial x}-\frac{2}{q_{Q, e q}} \frac{d V}{d x} W_{Q, 0} \\
& \frac{\partial W_{Q, 2}}{\partial t}=-\frac{q_{Q, e q}}{2 m} \frac{\partial W_{Q, 3}}{\partial x}+\frac{q_{Q, e q}}{2 m} \frac{\partial}{\partial x}\left[\sigma_{3,1} W_{Q, 1}\right]-\frac{q_{Q, e q}}{2 m}\left(4+\sigma_{2,0}\right) \frac{\partial W_{Q, 1}}{\partial x}-\frac{4}{q_{Q, e q}} \frac{d V}{d x} W_{Q, 1} \\
& \frac{\partial W_{Q, 3}}{\partial t}=-\frac{q_{Q, e q}}{2 m} \frac{\partial W_{Q, 4}}{\partial x}+\frac{q_{Q, e q}}{2 m} \frac{\partial}{\partial x}\left[\sigma_{4,2} W_{Q, 2}\right]-\frac{q_{Q, e q}}{2 m}\left(6+\sigma_{3,1}\right) \frac{\partial W_{Q, 2}}{\partial x}-\frac{6}{q_{Q, e q}} \frac{d V}{d x} W_{Q, 2}
\end{aligned}
$$

and so on for $W_{Q, n}, n>3$, with initial conditions $W_{i n, Q, n}$. A posteriori, the hierarchy Equation (85)-(88) enables one to establish the check of consistency at equilibrium. In fact, all $W_{e q, n}=0$ for $n=1,2,3,4, \ldots$ and $\partial W_{e q, 0} / \partial t=0$ satisfy Equations (85), (87) and (88). Finally, Equation (86) is also fulfilled by $W_{e q, 0}=h_{Q, 0}$, by virtue of Equations (76) for $n=1$ and Equation (79). The computations for $n=3,4,5, \ldots$ become extremely difficult. An open question is whether one also gets a three-term hierarchy for any $\mathrm{n}$. The above checks of consistency are independent of the specific 
$a_{e q, 0}$ given in Equation (51). Notice that in the actual case Equation (47), one has $\epsilon_{1,0}=\epsilon_{2,1}=0$, while $\epsilon_{2,0} \neq 0$. In this case, Equation (71) allows to prove that Equation (75) is indeed satisfied by $W_{e q, 1}=W_{e q, 2}=0$ and $W_{e q, 0}=h_{Q, 0}$ rather directly. The general comments in Subsection 4.1 after Equation (44) continue to hold here. Our aim here was to just show that, when Equation (47) holds, alternative orthogonal polynomials exist yielding another non-equilibrium hierarchy. We shall not deal with the issue of long-time approximations for Equations (85)-(88).

\section{C. $W_{e q, \gamma}$, Orthogonal Polynomials Generated by It and Hierarchy, When Equations (54) and} (55) Hold

Upon replacing Equation (56) into Equations (54) and (55) with $\partial W_{e q, \gamma}(x, q) / \partial t=0$, a lengthy computation yields the following four-term linear recurrence relation for the $a_{e q, \gamma, n}$ 's $(n=0,1,2,3, \ldots)$ :

$$
\begin{array}{r}
-\frac{q_{Q, e q} 2(n+1)}{2 m}\left[\frac{d a_{e q, \gamma, n+1}}{d x}-\frac{m \omega^{2} x}{\alpha} a_{e q, \gamma, n+1}\right]-\frac{q_{Q, e q}}{2 m}\left[\frac{d a_{e q, \gamma, n-1}}{d x}+\frac{1}{\alpha} \frac{d V_{1}}{d x} a_{e q, \gamma, n-1}\right] \\
+\frac{\hbar^{2}}{3 ! 2^{2}}\left[\frac{q_{Q, e q}}{2 m \alpha}\right]^{3} \frac{d^{3} V_{1}}{d x^{3}} a_{e q, \gamma, n-3}-\frac{\gamma n}{2} a_{e q, \gamma, n}+\gamma \frac{\alpha}{2 m \omega^{2}}\left[\frac{\partial}{\partial x}-\frac{m \omega^{2} x}{\alpha}\right] \frac{\partial}{\partial x} a_{e q, \gamma, n}=0
\end{array}
$$

with $a_{e q, \gamma,-1}=a_{e q, \gamma,-2}=a_{e q, \gamma,-3}=0$. We shall impose that all $a_{e q, \gamma, n}(x)$ 's, $n=0,1,2,3, \ldots$, do not diverge exponentially as $|x| \rightarrow+\infty$. With the choice $a_{e q, \gamma, 0}=a_{e q, 0}$ made in Subsection 6.1, all $a_{e q, \gamma, n}(x)$ 's, $n=1,2,3,4, \ldots$, are determined recurrently in terms of $a_{e q, \gamma, 0}(x)$, through Equation (89), through the obvious generalizations of Equations (77) and (78). Notice that $a_{e q, \gamma, n}(x) \neq 0$ for odd $n^{\prime}$ $\left(n^{\prime}=1,3,5, \ldots\right)$. For $g=0$, with nonvanishing $\beta$, $\hbar$ and $\gamma, a_{e q, \gamma, n}(x)=0$ for $n=1,2,3,4, \ldots$ while $a_{e q, \gamma, 0}(x)$ is X-independent.

Let us outline an attempt at dealing with off-equilibrium situations. By generalizing Equation (56), we shall expand the non-equilibrium Wigner function [fulfilling Equation (54)] in terms of the $H_{n}$ 's as:

$$
\begin{aligned}
W & =W_{e q}^{(0)} \sum_{n=0}^{+\infty} a_{\gamma, n} H_{n}\left(\frac{q}{q_{Q, e q}}\right) \\
a_{\gamma, n} & =\frac{\exp \left[+\frac{m \omega^{2} x^{2}}{2 \alpha}\right]}{\pi^{1 / 2} 2^{n} n !} \int_{-\infty}^{+\infty} \frac{d q}{q_{Q, e q}} H_{n}\left(q / q_{Q, e q}\right) W(x, q ; t)
\end{aligned}
$$

The $a_{\gamma, n}$ 's are the non-equilibrium moments of $W(x, q ; t)$ determined by the $H_{n}$ 's. We shall transform Equations (54) and (55) into a linear hierarchy for the $a_{\gamma, n}$ 's. For that purpose, we multiply Equation (54) by $H_{n}\left(q / q_{Q, e q}\right) / q_{Q, e q}$, integrate over $q$ and operate, so as to express the resulting equation solely in terms of the $a_{\gamma, n^{\prime}}$ 's. One finds the following four-term linear hierarchy:

$$
\begin{aligned}
\frac{\partial a_{\gamma, n}}{\partial t}= & -\frac{q_{Q, e q} 2(n+1)}{2 m}\left[\frac{d a_{\gamma, n+1}}{d x}-\frac{m \omega^{2} x}{\alpha} a_{\gamma, n+1}\right]-\frac{q_{Q, e q}}{2 m}\left[\frac{d a_{\gamma, n-1}}{d x}+\frac{1}{\alpha} \frac{d V_{1}}{d x} a_{\gamma, n-1}\right] \\
& +\frac{\hbar^{2}}{3 ! 2^{2}}\left[\frac{q_{Q, e q}}{2 m \alpha}\right]^{3} \frac{d^{3} V_{1}}{d x^{3}} a_{\gamma, n-3}-\frac{\gamma n}{2} a_{\gamma, n}+\gamma \frac{\alpha}{2 m \omega^{2}}\left[\frac{\partial}{\partial x}-\frac{m \omega^{2} x}{\alpha}\right] \frac{\partial}{\partial x} a_{\gamma, n}
\end{aligned}
$$

with $a_{\gamma,-1}=a_{\gamma,-2}=a_{\gamma,-3}=0$. One could argue that $W(x, q ; t)-W_{e q}(x, q)$ be the smaller (and eventually tend to zero), the larger $t$ is (at least, for some suitable class of initial non-equilibrium conditions). Then, one would argue that $a_{\gamma, n}$ would approach, somehow, $a_{e q, \gamma, n}$ which, as seen above, are non-vanishing if $g \neq 0$. Then, for long $t(>0)$, all $a_{\gamma, n}, n=1,2,3,4, \ldots$, would be non-vanishing as 
well, which would make the analysis rather difficult, and the argument may be not regarded as definitive. However, if $g=0$ all $a_{e q, \gamma, n}$ vanish for $n=1,2,3,4, \ldots$, so that the above argument is, due to the actual inclusion of dissipation $(\gamma \neq 0$ ), fully correct (and entirely convincing, compared to the loose one given previously in Subsection 4.1). Except for the choice made in Equations (47), (48) and (49), the expansion of the non-equilibrium $W$ in terms of standard Hermite polynomials made in Equation (90) is entirely analogous to the one employed in the very extensive work in [35]. The above difficulty that all $a_{\gamma, n}, n=1,2,3,4, \ldots$, be non-vanishing is bypassed based upon Equation (57).

The coefficients $\sigma_{\gamma, n, n-j}$ in Equation (58) depend on $x, \beta$ and $\gamma$ : they are determined recurrently through Equation (57). One important difference compared to Equation (81) is the actual recurrence relation:

$$
\left(2 y+B_{\gamma, n}\right) H_{Q, \gamma, n}(y)=H_{Q, \gamma, n+1}(y)+C_{\gamma, n} H_{Q, n-1}(y)
$$

for $n=0,1,2, \ldots$, with $C_{0} \equiv 0$. Here, one has $B_{\gamma, n} \neq 0$, as $\gamma \neq 0$. For instance: $B_{\gamma, 0}=\sigma_{\gamma, 1,0}$.

The lowest two equations in the recurrence for the new non-equilibrium moments $W_{\gamma, n}$ read:

$$
\begin{aligned}
\frac{\partial W_{\gamma, 0}}{\partial t}= & -\frac{q_{Q, e q}}{2 m} \frac{\partial W_{\gamma, 1}}{\partial x}+\frac{q_{Q, e q}}{2 m} \frac{\partial\left(\sigma_{\gamma, 1,0} W_{\gamma, 0}\right)}{\partial x}+\frac{\gamma}{2}\left[1+x \frac{\partial}{\partial x}\right] W_{\gamma, 0}+\gamma \frac{\alpha}{2 m \omega^{2}} \frac{\partial^{2} W_{\gamma, 0}}{\partial x^{2}} \\
\frac{\partial W_{\gamma, 1}}{\partial t}= & -\frac{q_{Q, e q}}{2 m}\left[\frac{\partial W_{\gamma, 2}}{\partial x}-\frac{\partial\left(\sigma_{\gamma, 2,1} W_{\gamma, 1}\right)}{\partial x}+\frac{\partial\left(\left(\sigma_{\gamma, 2,1} \sigma_{\gamma, 1,0}-\sigma_{\gamma, 2,0}+2\right) W_{\gamma, 0}\right)}{\partial x}\right. \\
& \left.+\sigma_{\gamma, 1,0} \frac{\partial\left(W_{\gamma, 1}-\sigma_{\gamma, 1,0} W_{\gamma, 0}\right)}{\partial x}\right]-\frac{2}{q_{Q, e q}} \frac{d V}{d x} W_{\gamma, 0} \\
& +\frac{\gamma}{2}\left[x \frac{\partial W_{\gamma, 1}}{\partial x}-x\left(\frac{\partial \sigma_{\gamma, 1,0}}{\partial x}\right) W_{\gamma, 0}+\sigma_{\gamma, 1,0} W_{\gamma, 0}\right] \\
& +\gamma \frac{\alpha}{2 m \omega^{2}}\left[\frac{\partial^{2} W_{\gamma, 1}}{\partial x^{2}}-2 \frac{\partial \sigma_{\gamma, 1,0}}{\partial x} \frac{\partial W_{\gamma, 0}}{\partial x}-\left(\frac{\partial^{2} \sigma_{\gamma, 1,0}}{\partial x^{2}}\right) W_{\gamma, 0}\right]
\end{aligned}
$$

At equilibrium, Equation (94) is satisfied by $W_{e q, \gamma, 1}=0$ and $W_{e q, \gamma, 0}=\pi^{1 / 2} \exp \left[-\frac{1}{\alpha} \frac{m \omega^{2} x^{2}}{2}\right] a_{e q, \gamma, 0}$, consistently. The above check of consistency is independent of the specific $a_{e q, \gamma, 0}$ selected above. We shall not delve into long-time approximations for Equations (94) and (95).

\section{Eigen-Operators of $H$ for $V_{1} \neq 0$}

An exact formal representation for Lindblad's operators is known, which has provided certain general properties for them [29]. On the other hand, it has not been easy for us to use that representation to get approximate or explicit expressions for Lindblad's operators in a direct way, when Equation (47) holds. Then, there may be a conceptual interest in investigating how could Lindblad's operators be constructed through alternative approximation procedures, at least when Equation (47) holds with $V_{1} \neq 0$, through Equations (62) and (63) (with $\omega^{\prime} \neq \omega$ ). Such a study will be undertaken below, in outline. We shall search for $A^{+}\left(\omega^{\prime}\right)$ as the formal operator series:

$$
\begin{aligned}
A^{+}\left(\omega^{\prime}\right)= & \alpha_{1,0} a+\alpha_{0,1} a^{+}+\alpha_{2,0} a^{2}+\alpha_{0,2}\left(a^{+}\right)^{2}+\alpha_{1,1} a^{+} a \\
& +\alpha_{3,0} a^{3}+\alpha_{0,3}\left(a^{+}\right)^{3}+\alpha_{1,2}\left(a^{+}\right)^{2} a+\alpha_{2,1} a^{+} a^{2}+\alpha_{4,0} a^{4} \\
& +\alpha_{0,4}\left(a^{+}\right)^{4}+\alpha_{1,3}\left(a^{+}\right)^{3} a+\alpha_{3,1} a^{+} a^{3}+\alpha_{2,2}\left(a^{+}\right)^{2} a^{2}+\cdots
\end{aligned}
$$


all $\alpha_{l, l^{\prime}}$ 's $\left(l, l^{\prime}=0,1,2,3, \ldots\right)$ being unknown coefficients. If the series Equation (96) fulfills Equation (63), then the coefficients of the successive operators $\left(a^{+}\right)^{l} a^{l^{\prime}}\left(l, l^{\prime}=0,1,2,3, \ldots\right)$ should match at both sides of Equation (63). To the order of truncation implied by the terms displayed in Equation (96), $\omega^{\prime}$ and the non-vanishing coefficients $\alpha_{l, l^{\prime}}$ are the solution of the following homogeneous linear system (corresponding to the matching of the coefficients of $\left(a^{+}\right)^{0} a^{0},\left(a^{+}\right)^{0} a, a^{+} a^{0},\left(a^{+}\right)^{0} a^{2}$, $\left(a^{+}\right)^{2} a^{0},\left(a^{+}\right) a$, and so on):

$$
\begin{aligned}
24 g_{1}\left(\alpha_{0,4}-\alpha_{4,0}\right)+12 g_{1}\left(\alpha_{0,2}-\alpha_{2,0}\right) & =0 \\
-\hbar \omega \alpha_{1,0}+24 g_{1} \alpha_{0,3}+12 g_{1}\left(\alpha_{0,1}+\alpha_{1,2}-3 \alpha_{3,0}\right)-12 g_{1} \alpha_{1,0} & =\hbar \omega^{\prime} \alpha_{1,0} \\
\hbar \omega \alpha_{0,1}-24 g_{1} \alpha_{3,0}+12 g_{1}\left(-\alpha_{1,0}-\alpha_{2,1}+3 \alpha_{0,3}\right)+12 g_{1} \alpha_{0,1} & =\hbar \omega^{\prime} \alpha_{0,1} \\
-2 \hbar \omega \alpha_{2,0}+12 g_{1} \alpha_{0,2}+24 g_{1} \alpha_{1,3}+12 g_{1}\left(\alpha_{1,1}+\alpha_{2,2}-6 \alpha_{4,0}\right)-g_{1} 6 \alpha_{2,0} & =\hbar \omega^{\prime} \alpha_{2,0} \\
2 \hbar \omega \alpha_{0,2}-12 g_{1} \alpha_{2,0}-24 g_{1} \alpha_{3,1}+12 g_{1}\left(-\alpha_{1,1}-\alpha_{2,2}+6 \alpha_{0,4}\right)+g_{1} 6 \alpha_{0,2} & =\hbar \omega^{\prime} \alpha_{0,2} \\
96 g_{1}\left(\alpha_{0,4}-\alpha_{4,0}\right)+g_{1} 12\left(4 \alpha_{0,2}-4 \alpha_{2,0}+\alpha_{1,3}-\alpha_{3,1}\right) & =\hbar \omega^{\prime} \alpha_{1,1} \\
-3 \hbar \omega \alpha_{3,0}+4 g_{1}\left(\alpha_{0,1}+3 \alpha_{1,2}\right)+4 g_{1}\left(3 \alpha_{2,1}-\alpha_{1,0}\right)-15 g_{1} \alpha_{3,0} & =\hbar \omega^{\prime} \alpha_{3,0} \\
3 \hbar \omega \alpha_{0,3}-4 g_{1}\left(\alpha_{1,0}-3 \alpha_{2,1}\right)+4 g_{1}\left(-3 \alpha_{1,2}+\alpha_{0,1}\right)+15 g_{1} \alpha_{0,3} & =\hbar \omega^{\prime} \alpha_{0,3} \\
-\hbar \omega \alpha_{2,1}+36 g_{1} \alpha_{0,3}+g_{1}\left(-36 \alpha_{3,0}+24\left(\alpha_{1,2}+12 \alpha_{0,1}\right)+g_{1}\left(-4 \alpha_{2,1}-2 \alpha_{1,0}\right)\right. & =\hbar \omega^{\prime} \alpha_{2,1} \\
-4 \hbar \omega \alpha_{4,0}+4 g_{1}\left(\alpha_{1,1}+3 \alpha_{2,2}\right)+4 g_{1}\left(3 \alpha_{3,1}-2 \alpha_{2,0}\right)-36 g_{1} \alpha_{4,0} & =\hbar \omega^{\prime} \alpha_{4,0} \\
4 \hbar \omega \alpha_{0,4}+4 g_{1}\left(-\alpha_{1,1}-3 \alpha_{2,2}\right)+4 g_{1}\left(-3 \alpha_{1,3}+2 \alpha_{0,2}\right)+36 g_{1} \alpha_{0,4}, & =\hbar \omega^{\prime} \alpha_{0,4} \\
\hbar \omega \alpha_{1,2}-36 \alpha_{3,0}+g_{1}\left(36 \alpha_{0,3}-24\left(\alpha_{2,1}-12 \alpha_{1,0}\right)+g_{1}\left(4 \alpha_{1,2}+2 \alpha_{0,1}\right)\right. & =\hbar \omega^{\prime} \alpha_{1,2} \\
-2 \hbar \omega \alpha_{3,1}+8 g_{1} \alpha_{0,2}+36 g_{1} \alpha_{1,3}+g_{1}\left(24 \alpha_{2,2}-48 \alpha_{4,0}+8 \alpha_{1,1}\right)-g_{1} 10 \alpha_{3,1}-4 g_{1} \alpha_{2,0} & =\hbar \omega^{\prime} \alpha_{3,1} \\
2 \hbar \omega \alpha_{1,3}-8 g_{1} \alpha_{2,0}-36 g_{1} \alpha_{3,1}+g_{1}\left(-24 \alpha_{2,2}+48 \alpha_{0,4}-8 \alpha_{1,1}\right)+g_{1} 10 \alpha_{1,3}+4 g_{1} \alpha_{0,2} & =\hbar \omega^{\prime} \alpha_{1,3} \\
72 g_{1} \alpha_{0,4}-72 g_{1} \alpha_{4,0}+g_{1}\left(36 \alpha_{1,3}-36 \alpha_{3,1}+24 \alpha_{0,2}-24 \alpha_{2,0}\right) & =\hbar \omega^{\prime} \alpha_{2,2}
\end{aligned}
$$

Equations (97)-(111) are a homogeneous linear system of fifteen equations for the fourteen unknowns $\alpha_{l, l^{\prime}}$ 's, written explicitly in Equation (96). On the other hand, $\omega^{\prime}$ is also unknown. The analysis of Equations (97)-(111) leads to the following conclusions: (i) $\alpha_{2,0}=\alpha_{0,2}=\alpha_{1,1}=\alpha_{4,0}=\alpha_{0,4}=\alpha_{3,1}=$ $\alpha_{1,3}=\alpha_{2,2}=0$, so that the nine Equations (97), (100), (101), (102), (107), (108), (109), (110) and (111) are satisfied identically; (ii) the remaining six $\alpha_{1,0}, \alpha_{0,1}, \alpha_{3,0}, \alpha_{0,3}, \alpha_{2,1}$ and $\alpha_{1,2}$ are nonvanishing and fulfill the homogeneous linear system of six equations, Equations (98), (99), (103), (104), (106) and (105), provided that (iii) the determinant formed by the coefficients of the homogeneous linear system Equations (98), (99), (103), (104), (106) and (105) vanishes, which yields $\omega^{\prime}$. More specifically, the vanishing of the determinant in (iii) yields two solutions, $\omega^{\prime}>0$ and $\omega^{\prime}<0$ which, in turn, determine two solutions for the coefficients of the system Equations (98), (99), (103), (104), (106) and (105) and, hence, two operators $A^{+}\left(\omega^{\prime}\right)$ with $\omega^{\prime}>0$ and $A^{+}\left(\omega^{\prime}\right)$ with $\omega^{\prime}<0$. Let $g_{1} \rightarrow 0$. Then, for $\omega^{\prime}>0$, as $\omega^{\prime} \rightarrow \omega, A^{+}\left(\omega^{\prime}\right) \rightarrow a^{+}$. For $\omega^{\prime}<0$, as $\omega^{\prime} \rightarrow-\omega$, then $A^{+}\left(\omega^{\prime}\right) \rightarrow a$. The adjoint of the solution with $\omega^{\prime}>0$ yields $A\left(\omega^{\prime}\right)$. To the actual order of truncation, all that yields completely Lindblad's operators in Equation (65).

The above study indicates clearly how the inclusion of higher order terms with nonvanishing coefficients in the operator series Equation (96) proceeds at higher orders: $\alpha_{l, l^{\prime}}=0$ or $\neq 0$, if $l+l^{\prime}$ is even or odd, respectively. Thus, the next set of nonvanishing coefficients is: $\alpha_{5,0}, \alpha_{0,5}, \alpha_{4,1}, \alpha_{1,4}$, 
$\alpha_{3,2}$ and $\alpha_{2,3}$. In addition to the counterparts of Equations (98), (99), (103), (104), (106) and (110) (in which the new set of six nonvanishing coefficients also contribute), one has an additional subset of six equations determined by $\alpha_{5,0}, \alpha_{0,5}, \alpha_{4,1}, \alpha_{1,4}, \alpha_{3,2}$ and $\alpha_{2,3}$, which contain all twelve $\alpha_{l, l^{\prime}}$ 's. Altogether, one has a homogeneous linear system of $6+6=12$ equations for the nonvanishing $6+6=12 \alpha_{l, l}$ 's: the vanishing of its determinant yields $\omega^{\prime}(>0)$ and $\omega^{\prime}(<0)$ to the new order of truncation, and so on for higher orders of truncation.

(C) 2012 by the author; licensee MDPI, Basel, Switzerland. This article is an open access article distributed under the terms and conditions of the Creative Commons Attribution license (http://creativecommons.org/licenses/by/3.0/.) 